\title{
Coacervate-based Underwater Adhesives in Physiological Conditions
}

\author{
Mehdi Vahdati, Francisco J. Cedano-Serrano, Costantino Creton*, Dominique Hourdet* \\ Soft Matter Sciences and Engineering, ESPCI Paris, PSL University, Sorbonne University, \\ CNRS, F-75005 Paris, France \\ Email: costantino.creton@espci.fr, dominique.hourdet@espci.fr
}

\begin{abstract}
Soft underwater adhesives which can function in physiological environments are in high demand for biomedical applications. This study establishes a clear link between the composition and mechanical properties of complex coacervates from poly(2-acrylamido-2-methylpropane sulfonic acid) (PAMPS) and poly(N,N-[(dimethylamino) propyl] methacrylamide) (PMADAP) with degrees of polymerization $(D P)$ close to 100. Choosing such low DPs offers several advantages including low water contents corresponding to strong mechanical properties while remaining in the unentangled regime to allow injectability at high salt concentrations. Most importantly, this strategy favors the occurrence of the salt-induced sol-gel transition near physiological concentrations, where these materials form sticky hydrogels due to their viscoelastic dissipative nature. The fluid-like coacervate prepared at $0.75 \mathrm{M} \mathrm{NaCl}$ behaves as a soft adhesive when injected in physiological conditions. This adhesive satisfies a nontrivial tradeoff between injectability and final mechanical properties. Alternatively, the gel-like coacervate prepared at $0.1 \mathrm{M} \mathrm{NaCl}$ offers an instant-stick solution in physiological conditions with a remarkable

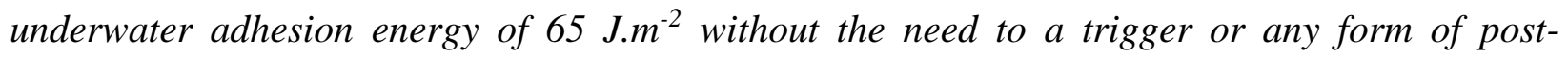
reinforcement. These coacervates mimic the behavior of soft adhesives in air and may be useful as biomedical adhesives.
\end{abstract}

Key Words: coacervates, underwater adhesion, Time Salt Superposition, sol-gel transition, biomedical adhesives, hydrogels

1. Introduction 
Associative liquid-liquid phase separations in aqueous media are ubiquitous in biological systems. ${ }^{1-3}$ From membrane-less compartmentalization of living cells to the delivery of the underwater glue of sandcastle worms, there are many instances where a fine thermodynamic balance between two liquid phases is absolutely critical to the desired function. ${ }^{2,4,5}$ Such phase separations are generically termed complex coacervation and can be driven by various molecular interactions (electrostatic, hydrophobic, H-bonding, etc.). ${ }^{6-8}$ Polyelectrolyte complex coacervation applies to the collapse of two oppositely-charged polyelectrolytes into a dense phase in equilibrium with a polymer-lean supernatant mostly composed of water. ${ }^{1,8,9}$

Polyelectrolyte complexation is generally believed to occur via an ion-exchange process involving the formation of macroion pairs between the oppositely charged residues of the polyelectrolytes upon the entropically favorable release of the counter ions into the supernatant. ${ }^{9-}$ ${ }^{11}$ According to this picture, the supernatant should contain a relatively higher salt concentration than the polymer-rich phase, corresponding to tie lines with a negative slope on a salt vs. polymer phase diagram. ${ }^{9,12-18}$ However, several studies have found the opposite (tie lines with a positive slope), ${ }^{9,19,20}$ while others have assumed equal salt concentrations in both phases (horizontal tie lines) for simplicity. ${ }^{10,21}$ This assumption is not always far from reality, since both experimentally measured and theoretically predicted differences in salt concentrations are quite small, especially at higher salt concentrations. , $9,17,22^{2}$

The macroion pairs between oppositely charged polyelectrolytes in a coacervate can be regarded as transient crosslinking points determining the viscoelastic properties of the material. In the unentangled regime, the polymer dynamics in the coacervate is qualitatively understood on the basis of the Sticky Rouse model with macroion pairs acting as stickers. Multiple associations, each made of $n$ consecutive stickers, form the binding sites between polymer chains which slow down the dynamics. ${ }^{23-26}$ For instance, in the works of Spruijt et. al. and Hamad. et. al., these clusters were assumed to be composed of $n=2$ and 5 pairs, respectively. ${ }^{24,25}$ In practice, $n$ consecutive stickers (forming a binding site) need to be opened all at once to allow local rearrangements leading to relaxation via motion on the order of a chain segment. ${ }^{24,27}$ Considered as an activated process, the lifetime of a sticker scales with the exponential of the activation energy to break the ionic contact, $E_{a}$, which is in turn a function of the square root of salt concentration as $E_{a}=A-B \sqrt{C_{s}}$; $A$ and $B$ being constants. Therefore, a general expression for the association lifetime is $\tau=$ $\tau_{0} \exp \left(\frac{n E_{a}}{k T}\right)$, with $\tau_{0}$ a microscopic time referred to as the attempt time. ${ }^{24}$ The association lifetime 
directly controls the polymer dynamics on longer time scales. For unentangled polymer solutions, the sticky Rouse model predicts the longest relaxation time as $\tau_{\max } \sim \tau$. $f^{2}$, where $f$ is the number of binding sites per chain.

The above description is simplistic in several ways. Firstly, it is valid under thermodynamic equilibrium, which is questionable at low salt concentrations. Another assumption of this theory is negligible changes in polymer volume fraction with salt concentration. Moreover, the macroions are taken as point charges in a continuum dielectric (Debye-Hückel approximation), and only local rearrangements, i.e. those on the order of a cluster, are taken into account. ${ }^{24,25,28}$ Charge connectivity, which has been shown to play an important role in coacervation, is thus mostly overlooked. ${ }^{9,15}$ Nevertheless, this model captures many features of complex coacervation as confirmed from experiments. ${ }^{29}$

For instance, the theory can be used to explain the doping effect of salt on the physical and mechanical properties of complex coacervates. ${ }^{6,26,30}$ Relatively low salt concentrations favor macroion pairing and lead to solid precipitates, called complexes, with long to infinite relaxation times. In fact, some of these complexes remain in a kinetically frozen state, never reaching thermodynamic equilibrium. This effect is more pronounced at higher polymer concentrations and molecular weights. Addition of salt weakens the complex by lowering the activation energy against dissociation. Beyond a certain salt concentration, a liquid-like coacervate characterized by relatively short relaxation times is obtained. ${ }^{25,28,29,31,32}$ The salt concentration at which this transition (from a solid complex to a liquid coacervate) occurs is determined by the molecular weight and monomer composition (hydrophilicity) of the polyelectrolytes, charge stoichiometry, as well as the type of salt. ${ }^{6,29,32,33}$ Beyond a critical salt concentration (CSC) where all the macroions are doped with small counter ions, phase separation is suppressed and a viscous, salty polyelectrolyte solution is obtained. ${ }^{21,32}$

Water content is a determining factor in the mechanical properties of soft materials such as hydrogels and soft tissues, in particular for applications where they are expected to remain stable and serve a certain function under immersed conditions for a given period of time. ${ }^{34}$ The equilibrium water content mainly depends on the hydrophobicity of the polyelectrolytes and the hydration level of the salt added. ${ }^{35}$ It is generally accepted that more hydrophobic polyelectrolytes form hard, solid precipitates with water contents below $40 \mathrm{wt} \%$ while more hydrophilic polyelectrolytes tend to form softer, liquid-like coacervates with water contents between 60-95 wt 
$\%{ }^{24,33}$ At the same concentration, weakly hydrated salts tend to swell a given complex coacervate to a larger degree than a strongly hydrated salt, following the Hofmeister's series. ${ }^{33,35}$ However, regardless of the system under study, it is crucial to bear in mind that the water content changes accordingly with the salt concentration, allowing access to a continuum of mechanical properties. ${ }^{6,33}$

The Sticky Rouse model is also helpful in understanding the underlying meaning of the socalled Time-Salt Superposition (TSS), first introduced by Spuijt et. al. ${ }^{25}$ They showed that the effect of salt on the relaxation spectra of complex coacervates is analogous to that of temperature on the dynamics of polymer melts. ${ }^{25,36}$ Since salt affects the relaxation times of all modes (ionic bonds) to the same extent, $\tau \propto \exp \left(A-B \sqrt{C_{S}}\right)$, addition or removal of salt shifts all the relaxation spectra towards higher or lower frequencies, respectively. ${ }^{25,28}$ This means the dynamic moduli of complex coacervates at different salt concentrations can be rescaled onto a master curve using saltdependent shift factors. The horizontal (temporal) shift factor, $a_{s}$, is a measure of how chain mobility, i.e. relaxation, is slowed by the macroion associations and is therefore strongly dependent on salt concentration. In contrast, the vertical shift factor, $b_{s}$, is only weakly dependent on added salt, but if anything, it is intuitively expected to decrease.

Since these first reports, TSS has been successfully applied to dynamic rheological data for other complex coacervates. ${ }^{24,26,28,29,31-33}$ The master curves obtained for complex coacervates from polyelectrolytes with varying degrees of polymerization (DPs) show that larger DPs give rise to a crossover in the dynamic moduli and the appearance of an elastic plateau at higher frequencies (above $1 / \tau$, where $\tau$ is the relaxation time of an ideal polymer chain). At low frequencies, however, a terminal flow behavior $\left(G^{\prime} \propto \omega^{2}, G^{\prime \prime} \propto \omega\right)$ is observed regardless of the molecular weight. ${ }^{24,28}$ At a given DP, coacervates prepared at higher salt concentrations contribute to the low-frequency, terminal part of the master curve, with more solid-like samples at lower salt concentrations providing the high frequency response. ${ }^{33}$

So far, most of the studies on the mechanical properties of complex coacervates have focused on relatively high molecular weight polyelectrolytes (DPs > 500). ${ }^{6,32,33,37,38}$ Lower mixing entropy at larger molecular weights generally leads to higher CSCs and stronger phase separations, with a more concentrated coacervate and a leaner supernatant. This corresponds to an expansion of the two-phase region of the phase diagram on the salt-polyelectrolyte plane. ${ }^{10}$ Intuitively, the sol-gel transition is also expected to move towards higher salt concentrations. Yet, coacervates based on 
lower molecular weight polyelectrolytes, which are expected to feature sol-gel transition at lower salt concentrations, remain understudied.

When placed in a low-salt medium for a sufficiently long period of time, a liquid-like coacervate is strengthened as salt diffuses out of it. ${ }^{39,40}$ Ideally, this would shift the relaxation spectra towards longer time scales and solidify the material if the sol-gel transition is crossed. This technique, known as salt switch, is potentially useful for a variety of purposes, such as controlling the porosity of polyelectrolyte complex-based hydrogels, membrane fabrication via phase inversion, and hardening an injectable coacervate-based underwater adhesive. ${ }^{38-41}$ The latter strategy is partly inspired by sandcastle worms which inject their glue components in a liquid state followed by insitu coacervation leading to a highly-wetting, low interfacial tension coacervate immiscible with seawater (note that, given the high ionic strength of seawater, the worm mainly takes advantage of an abrupt change in $\mathrm{pH}$ and ionic composition as well as oxidative post-curing of catechol moieties to set the glue). ${ }^{4,42}$

Given the relatively low ionic strength of the human body (with $0.137 \mathrm{M} \mathrm{NaCl}$ and traces of other salts), a salt switch into physiological conditions is potentially useful for biomedical applications such as embolization, sealing leaks, and tissue adhesion. However, two limiting factors, namely the tolerance threshold of human tissues to high salt concentrations and the slow nature of salt diffusion, undermine the viability of this strategy. To put things into perspective, solgel transitions with typical DPs in the literature (> 500) occur in the range of 0.6-1.3 M salt and in situ salt switch experiments take one to several hours. ${ }^{6,32,33,40}$

Dompé and coworkers recently proposed a novel macromolecular design to compensate for the slow salt switch of liquid-like coacervates by incorporating thermoresponsive grafts onto the polyelectrolyte backbones with DPs above $1000 .{ }^{37,40}$ This endowed the adhesive with a rapid initial hardening upon heating at body temperature, due to the formation of strong hydrophobic domains. With a sol-gel transition at around $0.5 \mathrm{M}$, formation of electrostatic interactions upon a salt switch into physiological salt concentrations was found to be notably larger than upon a thermal switch (to $37^{\circ} \mathrm{C}$ ) alone. One factor limiting the mechanical properties was the abundant water in the graft copolymer coacervates ( $90 \mathrm{wt} \%)$, regardless of salt concentration. By removing water from the coacervates via extrusion at $50{ }^{\circ} \mathrm{C}$, Dompé et al. managed to improve both the stiffness and the dissipative properties of the material and hence increase underwater adhesion by one order of magnitude. ${ }^{34}$ 
Here we propose simple model coacervates based on two oppositely charged polyacrylamides with DPs in the range of 100 , much lower than the focus of previous studies. ${ }^{6,24,28,32,33,40} \mathrm{In}$ particular, we benefit from such small DPs to tune the sol-gel transition around physiological salt concentrations, which can be exploited for the purpose of underwater adhesion. First, the composition and phase behavior of the coacervates are studied between the limiting cases of no added salt and a salt concentration above the CSC. We then use underwater linear rheology to characterize the sol-gel transition as well as to gain insights into the dynamic response of the coacervates using Time-Salt Superposition. Using a custom-built underwater probe tack setup, two of the coacervates are tested as underwater adhesives in physiological conditions. The first one is injectable at $0.75 \mathrm{M}$ salt and is tested after exposure to a salt switch ( $1 \mathrm{~h}$ in $0.1 \mathrm{M} \mathrm{NaCl})$, while the second one at $0.1 \mathrm{M}$ salt is already close to the critical gel point.

\section{Materials and Methods}

\subsection{Materials}

All the materials used in this work were purchased from Sigma-Aldrich. Potassium persulfate (KPS), 2-aminoethanethiol hydrochloride (AET-HCl), dicyclohexylcarbodiimide (DCCI), N-(3dimethylaminopropyl)-N'-ethylcarbodiimide hydrochloride (EDC), N-hydroxysuccinamide (NHS), acrylic acid (AA), and 1-methyl-2-pyrrolidinone (NMP) were used as received. The monomers 2-acrylamido-2-methylpropane sulfonic acid (AMPS) and $\mathrm{N}, \mathrm{N}-[($ dimethylamino) propyl]methacrylamide (MADAP) were also used without further purification. MADAP and AMPS come in basic $\left(-\mathrm{NR}_{3}\right)$ and acidic forms $\left(-\mathrm{SO}_{3} \mathrm{H}\right)$, respectively.

\subsection{Synthesis}

Details of the synthesis of the polyelectrolytes is given in the Supplementary Information (SI). Briefly, the two polyelectrolytes were synthesized similarly via free radical polymerization using a redox initiation system based on KPS and AET-HCl. ${ }^{43-45}$ The latter, which is a chain transfer agent, was used to 1) decrease the average degree of polymerization (DP) and 2) end-cap the polymers with a terminal amino moiety. A fraction of the amino-terminated polymers obtained were further modified with acrylic acid. These terminal double bonds, which can be later used to develop more sophisticated macromolecular architectures, are also very useful to characterize the average degree of polymerization (DP) of the polymers by ${ }^{1} \mathrm{H}-\mathrm{NMR}$ (see Figure S1 in the SI). The chemical structure of PAMPS and PMADAP are shown in Figure 1. The average DP of the 
polyanion, PAMPS, and the polycation, PMADAP, were determined to be 70 and 100 , respectively.

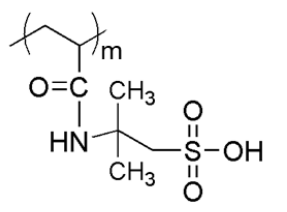

PAMPS $(m=70)$ poly(2-acrylamido-2methylpropane sulfonic acid)

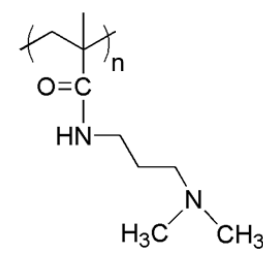

$\operatorname{PMADAP}(n=100)$

poly $(\mathrm{N}, \mathrm{N}-[($ dimethylamino $)$ propyl] methacrylamide)

Figure 1. The chemical structures and DPs of the polyelectrolytes used in the preparation of the complex coacervates. PAMPS is a strong polyanion $\left(P E^{-}\right)$while PMADAP is a weak polycation $\left(P E^{+}\right)$.

\subsection{Complex coacervation}

The polyelectrolyte complex coacervates were prepared in Falcon® tubes at a total polymer concentration of $0.094 \mathrm{M}$ in a total volume of $14 \mathrm{ml}$. The ratio of PMADAP $\left(\mathrm{PE}^{+}\right)$to PAMPS $\left(\mathrm{PE}^{-}\right)$was $53.2-46.8 \mathrm{~mol} \%$ such that the net charge is slightly positive. This corresponds to a total polymer weight of $247 \mathrm{mg}$ (in $14 \mathrm{ml}$ ) where the composition was 48.3-51.7 wt \% for PMADAPPAMPS. The samples were prepared at total nominal salt concentrations of $0.0,0.1,0.5,0.75,1.0$, 1.2 , and $1.35 \mathrm{M} \mathrm{NaCl}$, which will be used to distinguish the samples in the following. 


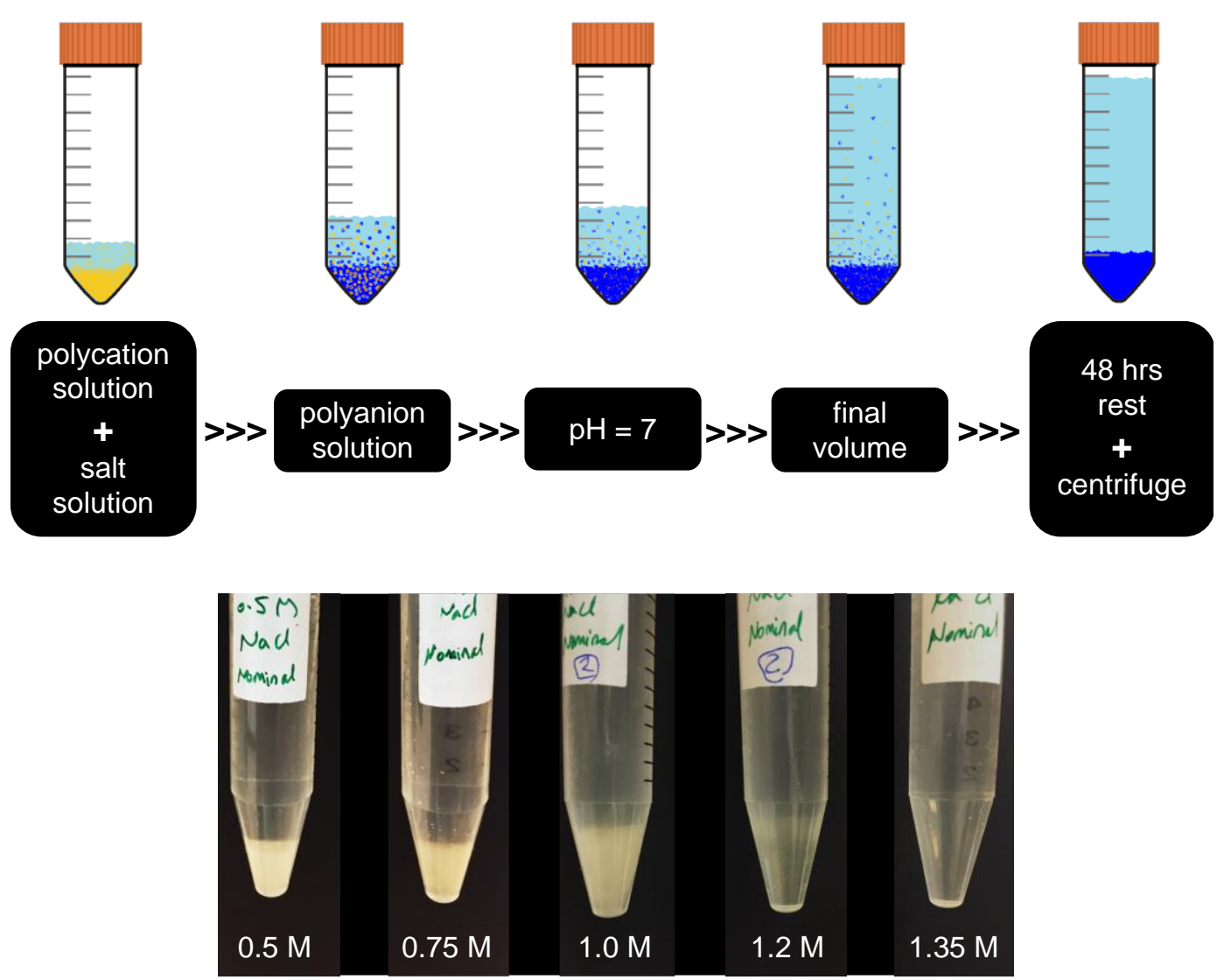

Figure 2. Schematic representation of different steps involved in the preparation of the complex coacervates along with pictures of some of the complex coacervates prepared as described above. The sample at $1.35 \mathrm{M}$ is already above the Critical Salt Concentration, where no macroscopic phase separation is observed.

Figure 2 schematizes the different steps of the preparation of the complex coacervates used in this work. Briefly, calculated volumes of separate stock solutions of each polyelectrolyte and $\mathrm{NaCl}$ (in Milli-Q water) were added into the Falcon ${ }^{\circledR}$ tubes, first mixing the salt and the polycation solutions followed by adding the polyanion solution. During this step, the total volume of acid/base added to each falcon tube was deduced from the amount of water required to reach the final volume of $14 \mathrm{ml}$. This was then added, and the samples were allowed to rest for $48-72 \mathrm{~h}$ to reach thermodynamic (pseudo)-equilibrium (it is known that complexes formed at very low salt concentrations may never reach thermodynamic equilibrium due to kinetic trapping effects). ${ }^{9}$ Finally, the samples were centrifuged and kept at ambient temperature before use. Pictures of some of the samples at equilibrium are shown at the bottom of Figure 2.

2.4. Thermogravimetric analysis (TGA) 
We used thermogravimetric analysis to characterize the composition of the coacervates, although other techniques such as conductivity measurements, UV-Vis spectroscopy, and labeling with isotopes are commonly used as well. ${ }^{15,30,32}$ TGA experiments in air were performed on an SDT Q600 (TA instruments) using standard platinum pans with a sufficiently high temperature tolerance for the purpose of this work. The sample, either a coacervate or a supernatant, was heated rapidly from room temperature to $110^{\circ} \mathrm{C}$, where it was held for $20 \mathrm{~min}$ to evaporate all the water, followed by heating to $1200{ }^{\circ} \mathrm{C}$ at $20^{\circ} \mathrm{C}$.min-1. The weight loss of the sample (in percentage) was recorded relative to the initial weight. Weight losses in the ranges of room temperature to $110{ }^{\circ} \mathrm{C}$ and 110 to $700{ }^{\circ} \mathrm{C}$ was assigned to water and polymer, respectively. Control experiments on each of the components (salt and polyelectrolytes solutions, see Figure S2 in the SI) showed that $\mathrm{NaCl}$ is removed completely by $1000{ }^{\circ} \mathrm{C}$, but residues of PAMPS (SO3 ${ }^{-}$) persist up to higher temperatures. This makes it difficult to determine the exact salt content for the coacervate phase where polymer concentration is much higher than in the supernatant. In the following, we will assume identical salt concentrations in both phases, although our data suggests slightly higher salt concentrations in the coacervate. In fact, recent literature suggests negligible differences in salt concentrations between the two phases, especially for samples prepared at higher salt concentrations. ${ }^{9,17,22}$ Typical mass loss curves from a coacervate and a supernatant are presented in Figure S3 in the SI. The details of follow-up calculations on the raw TGA data are given in the same section in the SI.

\subsection{Underwater linear rheology}

Underwater linear rheology was performed on a stress-controlled DHR-3 rheometer equipped with a PMMA cup installed around the bottom plate designed to measure in immersed conditions in different aqueous media. The dynamic behavior of each complex coacervate was studied in equilibrium, i.e. immersed in a medium at the same salt concentration as its supernatant, similar to the work of Hamad et. al. ${ }^{24}$ A cone-plate geometry was used, and the applied strain was limited to $1 \%$, within the linear regime for all the materials studied.

The samples at nominal salt concentrations of 0.1-1.0 M NaCl were tested using the same coneplate configuration. While the sample at $1.2 \mathrm{M}$ was too fluid to be properly characterized meaningfully on the DHR-3, the $0.0 \mathrm{M}$ sample was too stiff to be squeezed into the $53-\mu \mathrm{m}$ trim gap of the cone by the axial load cell of the rheometer $(50 \mathrm{~N})$. In the latter case, a flat-flat geometry 
with corrugated bottom and top plates and a gap of $400 \mu \mathrm{m}$ was used. For these samples, we will only use the data points that are physically meaningful.

Time-Salt Superposition (TSS) was performed using TRIOS software installed on the DHR-3 rheometer. Since TRIOS is only capable of performing Time-Temperature Superposition (TTS), we did the TSS manually by adapting its TTS function to our need. In order to validate the results, we performed the calculations manually as well, as detailed in the SI.

\subsection{Underwater probe tack}

The underwater probe tack experiments were performed on a custom-built setup designed by Sudre and coworkers in our laboratory. ${ }^{46}$ This setup has been used to study the underwater adherence of hard surfaces bearing polymer brushes ${ }^{46,47}$ and polymer thin films ${ }^{48}$ against macroscopic hydrogels. Recently, our team extended the use of this setup to study the underwater adhesion of different hard substrates against thermoresponsive complex coacervates. ${ }^{34,49}$ We used the procedure of Cedano-Serrano et. $\mathrm{al}^{48}$ to study underwater adhesion between our thermoresponsive complex coacervates and crosslinked thin films of PAA covalently bound to silicon wafers. These PAA thin films have a thickness of $\sim 255 \mathrm{~nm}$ at $\mathrm{pH} 7$ in the swollen state. ${ }^{48}$

Briefly, the setup consists of a chamber which can be filled with different aqueous media. Visual observation of the experiment is feasible through a transparent polycarbonate slide on the side of the setup. The chamber is mounted and fixed on an Instron machine (model 5565 for tensile and compression tests). The bottom of the chamber is shaped in the form of a sample trough to hold glass slides with a width of $2.5 \mathrm{~cm}$ as the bottom plate. For the top plate, also called the probe, a fresh PAA thin film was chemically grafted to a silicon wafer, itself attached to a cylindrical punch using a cyanoacrylate adhesive (Loctite ${ }^{\circledR} 495$, France). The mobile punch was connected to the crosshead of the Instron machine. The alignment of the probe against the glass slide to ensure a good contact was ensured in air while the PAA film was protected by Parafilm ${ }^{\circledR}{ }^{47,48}$

Figure 3 presents a schematic overview of the underwater probe tack setup and the experimental procedure adopted. All experiments were performed at room temperature, between 20 and $25^{\circ} \mathrm{C}$. Depending on the surface area of the probe, the appropriate amount of the coacervate was placed on the glass plate, using a Pasteur pipette for the liquid coacervate and a spatula for the gel-like sample. The probe was then lowered so that it was in full contact with the coacervate squeezed to an initial thickness $\left(h_{0}\right)$ of $500 \mu \mathrm{m}$ (Figure 3). Both samples were then immersed for $1 \mathrm{~h}$ in $0.1 \mathrm{M}$ $\mathrm{NaCl}$ at $\mathrm{pH}=7$, close to physiological salt concentration. The probe was detached from the sample 
at a given nominal strain rate $(\dot{\varepsilon})$ (as an example, $\dot{\varepsilon}=0.2 \mathrm{~s}^{-1}$ corresponds to a constant debonding velocity of $100 \mu \mathrm{m} . \mathrm{s}^{-1}$ ). The adhesive performance of the gel-like sample was also tested in Phosphate Buffered Saline (PBS), similarly after $1 \mathrm{~h}$ of immersion, as well as at different nominal strain rates. All experiments were repeated on at least 3 independently prepared samples.

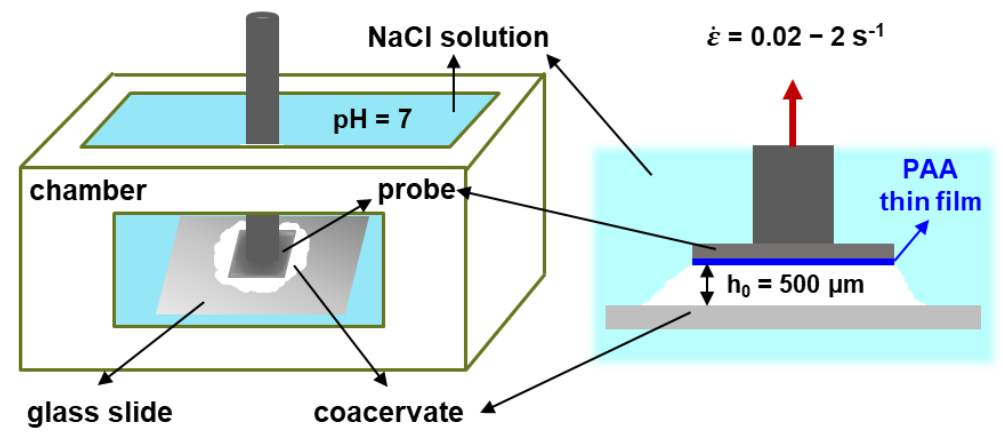

Figure 3. Schematic representation of the custom-made underwater probe tack setup for testing underwater adhesiveness of complex coacervates.

From the raw force $(F)$ and displacement $(h)$ data, nominal stress $(\sigma)$ and nominal strain $(\varepsilon)$ were calculated as:

$$
\sigma=\frac{F}{A_{0}}
$$

and

$$
\varepsilon=\frac{h-h_{0}}{h_{0}}
$$

Where $A_{0}$ is the surface area of the probe. The adhesion energy $\left(W_{a d h}\right)$ is directly proportional to the area under the stress-strain curve as:

$$
W_{a d h}=h_{0} \int_{0}^{\varepsilon_{\max }} \sigma \cdot d \varepsilon
$$

\section{Results}

\subsection{Composition and thermodynamics}

Figure 4 shows the composition of the supernatant and coacervate phases resulting from the complex coacervation process (below the CSC). The values were derived from TGA measurements on each phase in equilibrium (1 week after preparation). The TGA curves obtained for the coacervate and supernatant phases of the $0.5 \mathrm{M}$ sample are presented in Figure S3. As mentioned in the experimental section, the salt concentrations in the coacervate was assumed 
identical to that in the supernatant, where the sensitivity of the measurement was higher. These concentrations were found to be close to the intended (nominal) salt concentrations (see Table S1).

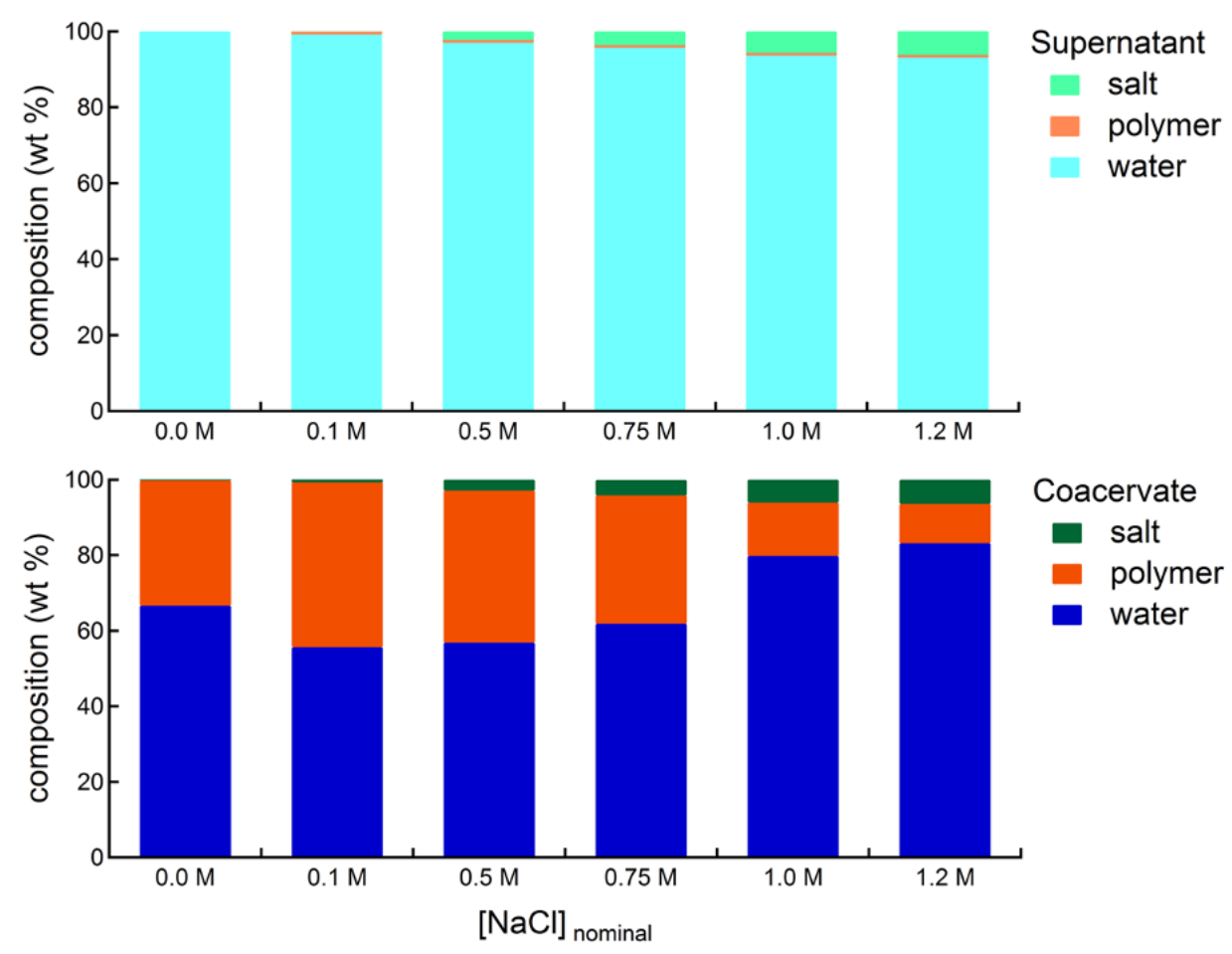

Figure 4. Composition of supernatants (top) and coacervates (bottom) for samples prepared at different nominal salt concentrations.

All the supernatants are almost entirely composed of water and salt with negligible concentrations of polyelectrolytes $(<0.6 \mathrm{wt} \%)$. The coacervates, on the other hand, contain up to $44 \mathrm{wt} \%$ polymer (in the case of the $0.1 \mathrm{M}$ sample), which decreases as more salt is added. This in turn allows more water to swell the coacervate phase (see Figure S4), which has important implications for the mechanical properties, as will be shown later. Such water contents (55-85 wt $\%)$ are characteristic of relatively hydrophilic polyelectrolytes. ${ }^{24,33}$ Above the CSC where all the electrostatic interactions are strongly screened, complexation does not occur and a viscous salty polyelectrolyte solution is obtained. ${ }^{1,6}$ This concentration falls between 1.2 and $1.37 \mathrm{M} \mathrm{NaCl}$ in this case.

The coacervate formed in the absence of salt was found more swollen (containing more water and less polymer) compared to the coacervates containing up to $0.75 \mathrm{M}$ salt. We believe this is because polyelectrolyte complexes formed at very low salt concentrations are often kinetically 
trapped, non-equilibrium materials..$^{9,50,51}$ For clarity, this does not imply that their composition and properties are not reproducible. The dynamics of strong associations formed at such low salt concentrations can be on the order of tens of years and hence beyond observable time scales. Another way of regarding this would be that the strong phase separation in the absence of salt causes local heterogeneities with regions of lower polymer chain density, leading to chains that are not fully complexed (i.e. certain chain segments remain charged with free counter ions) and/or weaker macroion pairs due to steric and conformational hindrance on local length scales. ${ }^{9}$

The salt entering the coacervate phase can either break macroion pairs $P E^{+} P E^{-}$into charges compensated by counter ions or remains as co-ions, i.e. not associated with the polyelectrolytes. The former case is described by the following equilibrium:

$$
P E^{+} P E^{-}+N a^{+}+C l^{-} \leftrightarrows P E^{+} \mathrm{Cl}^{-}+P E^{-} \mathrm{Na}^{+}
$$

The total salt concentration in the coacervate is then given by:

$$
[\mathrm{NaCl}]=\frac{\left[\mathrm{Na}^{+}\right]_{\text {count }}+\left[\mathrm{Cl}^{-}\right]_{\text {count }}}{2}+\frac{\left[\mathrm{Na}^{+}\right]_{\mathrm{CO}-\mathrm{ion}}+\left[\mathrm{Cl}^{-}\right]_{\mathrm{co}-\mathrm{ion}}}{2}
$$

The following discussion assumes equimolar salt concentrations in both supernatant and coacervate phases under equilibrium. This corresponds to ideal complexation driven by entropy alone. ${ }^{8}$ Two important parameters can be determined from this equilibrium.

1) the molar ratio, $r$, between salts and polyelectrolytes in the coacervate phase:

$$
r=\frac{\left[\mathrm{Na}^{+}\right]+\left[\mathrm{Cl}^{-}\right]}{\left[\mathrm{PE}^{+}\right]+\left[\mathrm{PE}^{-}\right]}=\frac{[\mathrm{NaCl}]}{[\mathrm{PE}]}
$$

with $[P E]$ the molar concentration of polyelectrolytes in the coacervate phase, such that $\left[P E^{+}\right]$ $=\left[\mathrm{PE}^{-}\right]=[\mathrm{PE}]$. Note also that $\left[\mathrm{Na}^{+}\right]=\left[\mathrm{Cl}^{-}\right]=[\mathrm{NaCl}]$.

2) the doping level, $y$, corresponding to the fraction of polyelectrolyte compensated by counter ions, i.e. the total number of counter ions to the total number of macroions: ${ }^{6,30,35}$

$$
y=\frac{\left[\mathrm{Na}^{+}\right]_{\text {count }}+\left[\mathrm{Cl}^{-}\right]_{\text {count }}}{\left[\mathrm{PE}^{+}\right]+\left[\mathrm{PE}^{-}\right]}=r \cdot \frac{\left[\mathrm{Na}^{+}\right]_{\text {count }}+\left[\mathrm{Cl}^{-}\right]_{\text {count }}}{\left[\mathrm{Na}^{+}\right]+\left[\mathrm{Cl}^{-}\right]}
$$

Using the equilibrium constant between paired and unpaired polyelectrolytes (see calculations based on equilibrium in the SI), the doping level can be extrapolated from $r$ as:

$$
y=\frac{r}{1+r}
$$

The doping level is expected to increase with salt concentration from 0 at no added salt to 1 at the CSC. Experimentally, it has been shown that this variation is linear, the slope being a quantitative measure of the efficiency of the salt at breaking macroion pairs $\left(P E^{+} P E^{-}\right) .{ }^{8,52}$ Therefore, a linear extrapolation to $\mathrm{y}=1$, i.e. full doping, is generally a good approximation of the 
CSC. Figure 5 plots the values of $r$ measured experimentally and $y$ calculated from equation (8) versus the measured salt concentration in the coacervate phase for samples below the CSC. The dashed line is a linear fit to $y$ values extrapolated to $y=1$, where the arrow marks the predicted CSC to be around 1.4 M NaCl. We experimentally found the CSC occurs just below $1.37 \mathrm{M} \mathrm{NaCl}$.

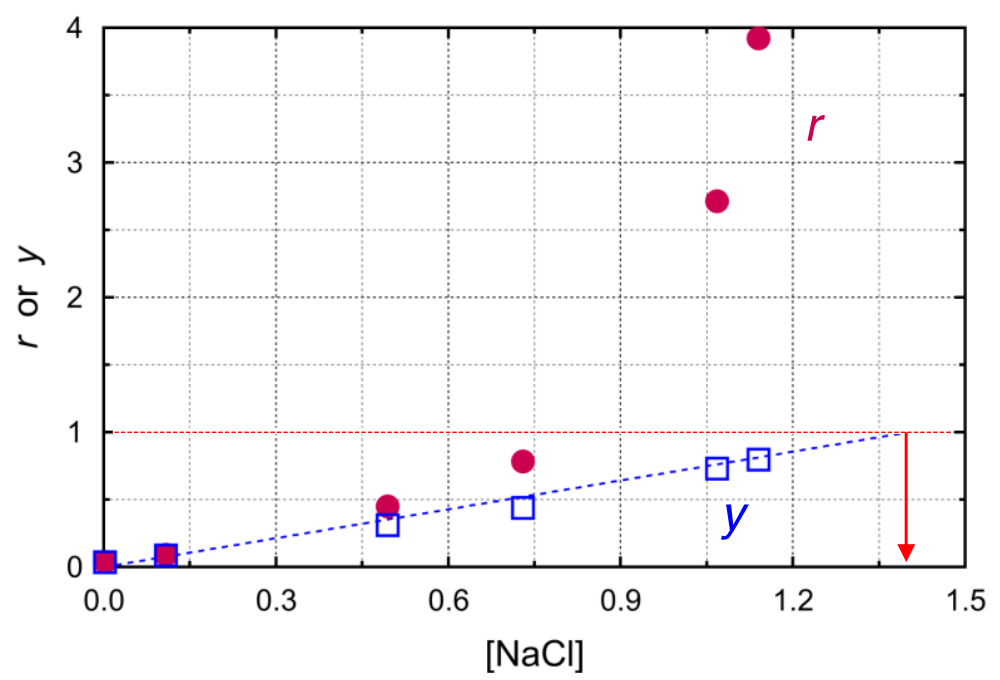

Figure 5. The doping level, $y$, and the molar ratio between salts and polyelectrolytes in the coacervate phase, $r$. Values of $r$ and $y$ were obtained from TGA data and calculated from equation (8), respectively. The arrow marks the CSC as predicted from $y=1$.

As seen in Figure 5, $r$ starts to deviate from $y$ around $0.5 \mathrm{M} \mathrm{NaCl}$. This signifies that below this point (at $y \cong r \ll 1$ ), most of the salt entering the coacervate acts as counterions breaking macroion pairs. The widening gap between $r$ and $y$ at higher salt concentrations marks the growing fraction of the salt ending up as (free) co-ions. This observation is in qualitative agreement with the results of Schlenoff's group who investigated various polyelectrolyte pairs of various DPs with different salts. ${ }^{6,8}$ This effect is attributed to the fixed charges of the complex coacervate that generate a Donnan potential excluding mobile counter ions from the rich polymer phase. This phenomenon is particularly efficient in low ionic strength environments, where mainly ions forming ion-paired with the polyelectrolyte are expected in the concentrated phase. This selectivity is no longer valid for sufficiently high salt concentrations and mobile ions can diffuse into the coacervate. This condition known as Donnan breakdown is typically expected when the salt concentration exceeds the concentration of fixed charges. $6,8,53$ 
Figure 6 summarizes the above results in a phase diagram plotting measured concentrations of salt and polyelectrolytes in preparation (hollow circles at $\left[\mathrm{PE}^{+}\right]+\left[\mathrm{PE}^{-}\right]=0.094 \mathrm{M}$ ) and in equilibrium. The filled circles on the left and right branches of the phase diagram mark the concentrations (calculated from TGA data) in the supernatant and coacervate phases, respectively. Horizontal tie lines on the phase diagram reflect the assumption of identical equilibrium salt concentrations in both phases in equilibrium, as mentioned earlier.

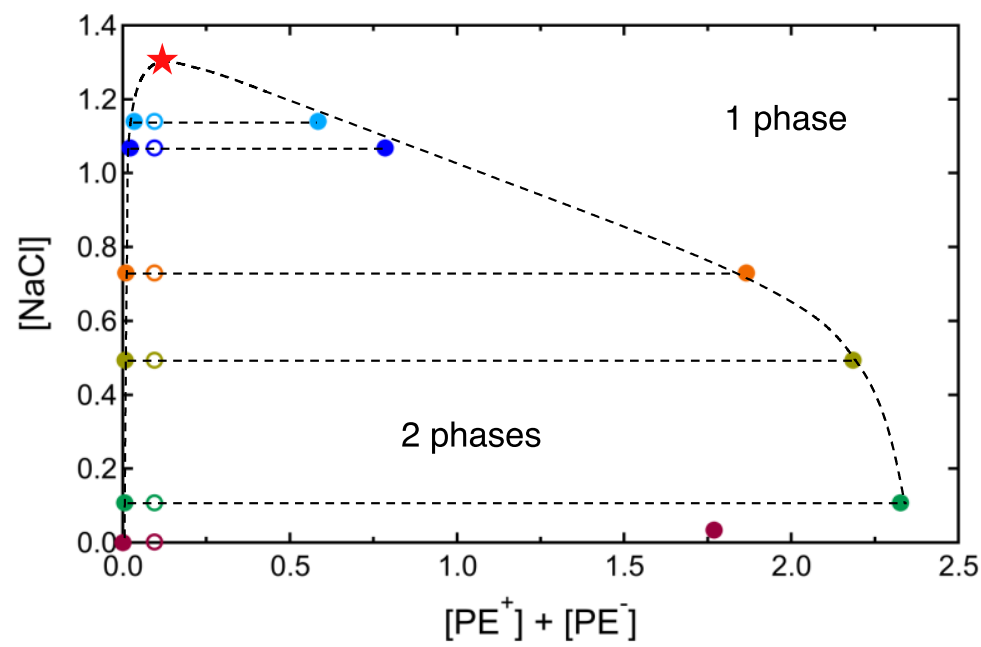

Figure 6. Phase diagram for complex coacervates prepared at different [ $\mathrm{NaCl}]$ concentrations between $0.0 \mathrm{M}$ and 1.2 M. The hollow circles mark preparation concentrations, while the solid circles to their left and right correspond to concentrations in the supernatant and the coacervate, respectively. The estimated critical salt concentration is marked with the red star. The dashed lines are guides to the eye.

While the concentration of polyelectrolytes in the supernatant remains negligible (below 0.03 $\mathrm{M})$, the coacervate phase is considerably richer in polymer and very sensitive to the presence of salt. Below 1.0 M NaCl, the two-phase region is quite wide, indicating a strong phase separation. The two-phase region then shrinks at higher salt contents, reaching the critical salt concentration (CSC) by $1.37 \mathrm{M} \mathrm{NaCl}$ (roughly marked by the red star). The bottom of the phase diagram (corresponding to the sample prepared without addition of salt) shows apparently enhanced miscibility, but this is simply due to the much stronger interactions leading to a kinetically frozen (out of equilibrium) microstructure, as explained above.

3.2. Underwater rheology 
Knowing the composition of the complex coacervates, we characterized their rheological behavior under equilibrium, i.e. in a medium at the same $\mathrm{pH}$ and salt concentration as their supernatant. The frequency-dependence of their moduli is then used to determine the salt concentration at which the complex coacervate experiences a sol-gel transition (going from a fluid coacervate to a complex with solid-like properties). We also explore the applicability of Time-Salt Superposition in order to expand the accessible dynamic behavior of the material to a larger frequency range and deepen our understanding of its nanostructure.

\subsubsection{Sol-gel transition}

Figure 7 A shows the frequency-dependence of the storage $\left(G^{\prime}\right)$ and loss $\left(G^{\prime \prime}\right)$ moduli for the coacervates prepared at different nominal salt concentrations. The corresponding changes in complex viscosity $\left(\eta^{*}\right)$ are presented in Figure S5 A. The coacervates prepared at 1.0 and $0.75 \mathrm{M}$ $\mathrm{NaCl}$ are in a fluid state $\left(G^{\prime \prime}>G^{\prime}\right)$. At lower salt concentrations, the materials become quite viscoelastic and begin to approach the gel point. The values of the loss and storage moduli, still quite dependent on frequency, remain close $\left(G^{\prime \prime} \approx G^{\prime}\right)$ over the entire range studied. Only in the extreme absence of salt $(0.0 \mathrm{M})$ does coacervation lead to a viscoelastic, solid-like $\left(G^{\prime}>G^{\prime \prime}\right)$ complex. The slightly higher moduli of the $0.0 \mathrm{M}$ sample compared to the $0.1 \mathrm{M}$ coacervate seems to be in contrast to the trend in water content (see Figure 4), but such a non-equilibrated complex is very likely to have a highly heterogenous nanostructure, with the seemingly higher water content trapped in the form of pockets of water. Nonetheless, the most interesting feature of this system is that it is on the verge of gelation-although still in the sol state- at physiological salt concentration. This has important implications for the underwater adhesiveness of these coacervates, as will be discussed later.

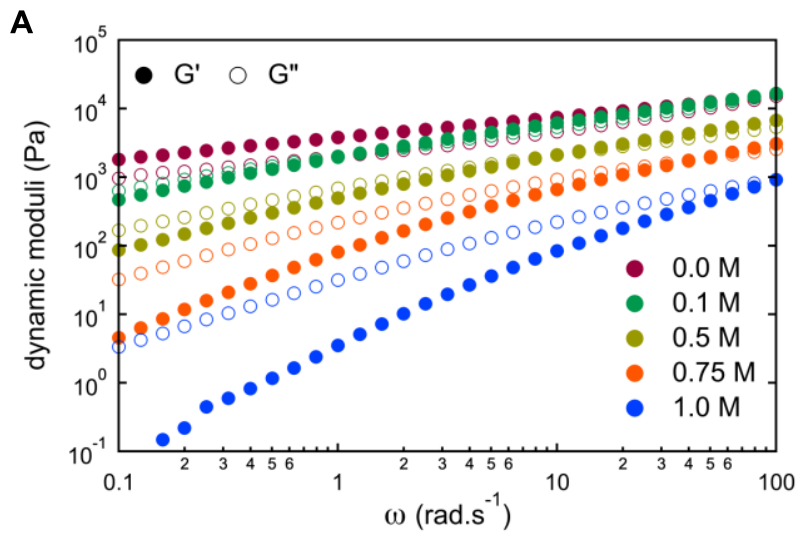

B

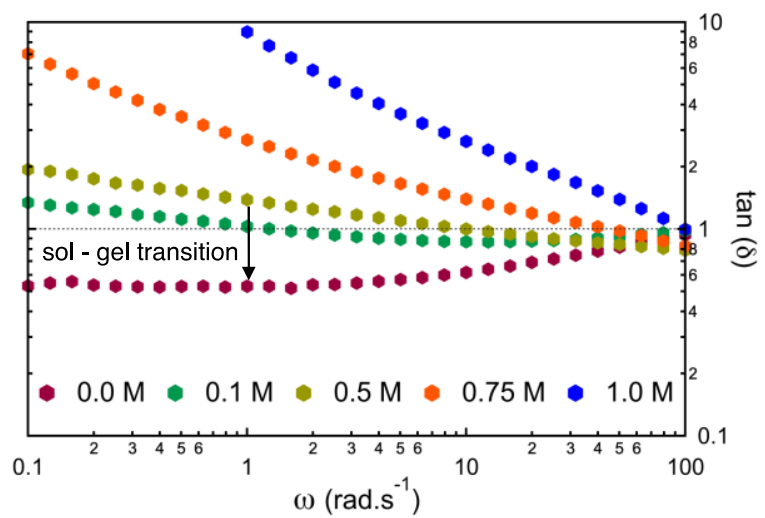


Figure 7. The dynamic response of the complex coacervates prepared at different salt concentrations represented in terms of the frequency-dependence of $(A)$ the storage $\left(G^{\prime}\right)$ and loss $(G$ ") moduli, and $(B)$ the tangent of phase angle, or $\tan (\delta)=G " / G$ '.

The above trends are clearly manifested in the change in the slope of $\tan (\delta)$ vs. frequency, presented in Figure 7 B. During a sol-gel transition, the slope of $\tan (\delta)$ changes from negative for liquid-like samples to nearly zero for critical gels and positive values for more solid-like gels. ${ }^{29,54}$ The relaxation modulus, $G(t)$, of any critical gel (i.e. one at the sol-gel transition point) is known to take a power-law form of: $:^{54,55}$

$$
G(t)=G_{0}\left(\frac{t}{\tau_{0}}\right)^{-s}
$$

where $0<s<1$ and $G_{0}$ is a measure of the stiffness of the critical gel in its unrelaxed state. Typically, soft critical gels are characterized by large values of $s(s \rightarrow 1)$, whereas stiff critical gels have smaller exponents $(s \rightarrow 0) .{ }^{54,55}$ To assess the stiffness of our critical gel, we can determine the power-law relaxation exponent ( $s$ in equation 9 ) based on the frequency-invariant phase angle as:

$$
s=\frac{2 \delta_{c}}{\pi}
$$

The closest sample to the actual critical gel where $\tan (\delta)$ is entirely independent of frequency is the $0.0 \mathrm{M}$ sample. With a critical $\tan \left(\delta_{c}\right) \cong 0.62$ (the average value of its $\tan (\delta)$ over $0.1-100$ rad. $\left.\mathrm{s}^{-1}\right)$, an exponent of $s=0.35$, characteristic of a stiff critical gel, is obtained. Liu et. al. found a similar power-law relaxation exponent for critical gels of PSS/PDADMAC complex coacervates with $\mathrm{KBr}$ as the salt. ${ }^{32}$ Since in their work the sol-gel transition occurred at $0.85 \mathrm{M} \mathrm{KBr}$, they were able to prepare more samples below the gel point, which allowed a more thorough analysis of the transition using the approach taken above.

The occurrence of the sol-gel transition close to the physiological salt concentration paves the road toward the design of viscoelastic underwater adhesives relying on nonspecific chemistry. In fact, sol-gel transitions previously reported in the literature fall outside the convenient range for biomedical applications, plausibly due to the large molecular weights used. ${ }^{6,32,33}$ In the following, we take advantage of the Time-Salt Superposition (TSS) principle to cast further light on this point. 


\subsubsection{Time-Salt Superposition (TSS)}

Figure 8 A shows the master curve obtained from applying a TSS procedure to the viscoelastic data of the complex coacervates (from Figure 7), i.e. by taking the $0.5 \mathrm{M}$ sample as the reference and shifting the data of the dynamic moduli along the horizontal and the vertical axes using the shift factors $a_{s}$ and $b_{s}$. Figure $8 \mathrm{~B}$ shows the values of these shift factors as a function of the measured (real) salt concentrations. The complex viscosity data and the corresponding master curve are presented in Figure S5 in the SI. Due to the non-equilibrium structure of the $0.0 \mathrm{M}$ coacervate, its viscoelastic data was not used in TSS. Although the spectrum of relaxation times of the coacervates varies with salt content, a master curve spanning more than 6 decades of frequency was readily obtained using mainly the terminal relaxation time (the horizontal shift factor, $\left.a_{s} \sim \tau_{\max }\right)$, since the vertical shift factor $\left(b_{s}\right)$ related to the corresponding modulus $\left(G\left(\tau_{\max }\right)\right)$ is only weakly dependent on salt concentration.

A

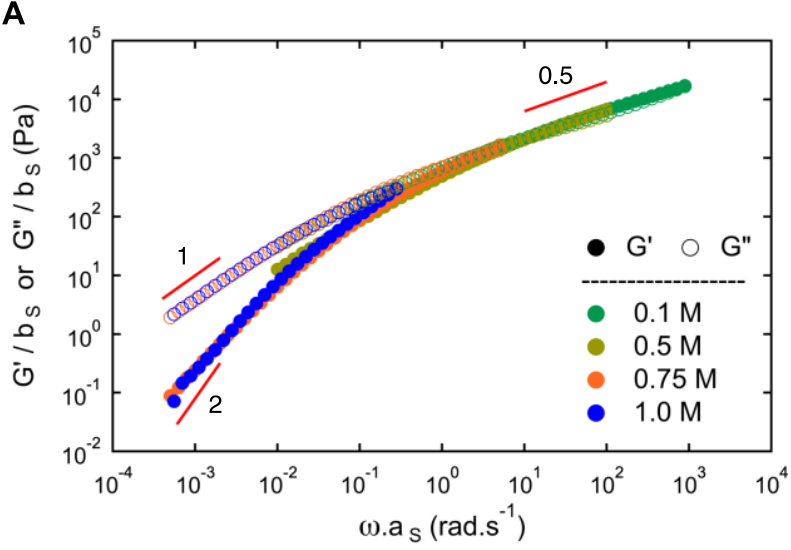

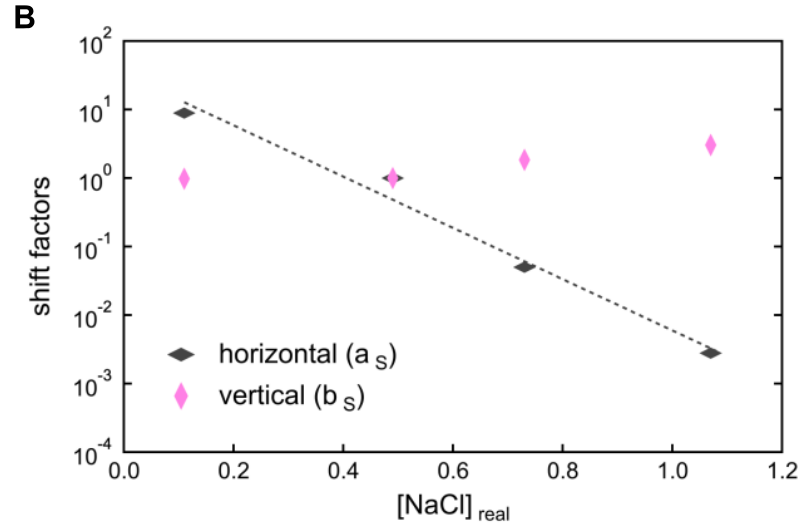

Figure 8. (A) rescaled viscoelastic data of the complex coacervates using Time-Salt Superposition and $(B)$ the shift factors, $a_{s}=\tau / \tau_{\text {ref }}$ and $b_{s}=G / G_{\text {ref }}$ calculated taking the 0.5 $M$ sample as the reference. The dashed line marks $\tau \propto \exp \left(A-B \cdot C_{S}\right), r^{2}=0.98$.

The dynamic response of our complex coacervates is typical of semi-dilute, unentangled polyelectrolyte solutions with a Rouse-like relaxation behavior: at intermediate frequencies $\left(1 / \tau_{R}<\omega<1 / \tau_{0}\right.$ where $\tau_{0}$ and $\tau_{R}$ are the relaxation times of the monomer and of the ideal polymer chain, respectively), the dynamic moduli scale with the square root of the angular frequency, $\omega^{0.5}$, while at low frequencies $\left(\omega<1 / \tau_{R}\right)$ the response is typical of a viscoelastic fluid $\left(G^{\prime \prime} \propto \omega\right.$ and $\left.G^{\prime} \propto \omega^{2}\right) .{ }^{28,31,36,56}$ Interestingly, Spruijt et. al. reported similar behaviors with 
hydrophilic coacervates of poly(acrylic acid) and poly(N,N-dimethylaminoethyl methacrylate) with DPs in the range of 50-150. ${ }^{28}$ Hamad et. al. found a qualitatively similar trend for master curves from non-matching complex coacervates with a polyanion, poly(isobutylene-alt-maleate sodium), of varying DPs $(65,475,1140$, and 2380) and a polycation, poly(diallyldimethylammonium chloride), having a DP around 1800. The master curve corresponding to the lowest molecular weight polyanion showed no sign of entanglements, i.e. no elastic plateau. ${ }^{24}$ By simultaneously applying Time-Salt and Time-Temperature superpositions between -5 to $45{ }^{\circ} \mathrm{C}$, Yang and coworkers detected no elastic plateau in coacervates of poly(methacrylic acid) and poly[3-(methacryloylamino) propyltrimethylammonium chloride] with DPs $\sim 200 .{ }^{26}$ These results seem to suggest that the DP of the polymer plays a more important role in controlling the mechanical properties of the coacervate compared to the chemistry of the monomers used. We believe this is at least true for systems at comparable water contents.

The sharp decrease of the longest relaxation time $\left(\tau_{\max } \sim a_{s}\right)$ with increasing salt concentration can be discussed in the framework of sticky polymer dynamics taking into account

$$
\tau_{\text {max }} \sim \tau \cdot f^{2} \sim \tau_{0} \exp \left(\frac{n E_{a}}{k T}\right) \cdot f^{2}
$$

with $n$ the number of consecutive stickers forming the binding sites, $E_{a}$ the activation energy of the dissociation process of a macroion pair and $f$ the number of binding sites per chain..$^{23,24,26}$ This relation qualitatively emphasizes that the addition of salt decreases the terminal relaxation time by reducing the activation energy for the dissociation of stickers as well as the number of macroion pairs between oppositely charged polyelectrolytes, affecting both $n$ and $f$. Ali and Prabhu as well as Yang et. al. have highlighted reduced polymer volume fraction as a third effect of added salt. ${ }^{26,57}$ This effect is seen in the deviation of $r$ (the molar ratio between salts and polyelectrolytes) from $y$ (the doping level) as well as the nonlinear increase in swelling at high salt concentrations as seen in Figure 5 and Figure S4, respectively. In general, the lower polymer volume fraction has a less significant contribution in decreasing the terminal relaxation time compared to the reductions in activation energy and the number of macroion pairs. ${ }^{25,28}$

Based on the sticky Rouse dynamics, the horizontal shift factor is expected to scale with $\exp \left(A-B \sqrt{C_{S}}\right)$. Many previous studies reported a good agreement between this theory and their experimental data. ${ }^{24,25,28,29,32,57}$ However, Marciel et. al. and Yang et. al. obtained better master 
curves by using an empirical horizontal shift factor that scaled exponentially with $A-B \cdot C_{S}$ and $A-B \cdot\left(C_{S}{ }^{6 / 5}\right)$, respectively. ${ }^{26,31}$ As shown in Figure $8 \mathrm{~B}$ (dashed line), we find a similar dependence as Marciel et. al. ${ }^{31}$ Such inconsistencies with the theory may arise from the simplistic assumptions of the model: Our samples may not reach thermodynamic equilibrium at low salt concentrations, there may be a broad relaxation time distribution in the course of the sol/gel transition and the variation with salt content of the polymer concentration in the coacervate phase was not taken into account.

In principle, the vertical shift factor $b_{s}=G / G_{\text {ref }}$ should decrease since the polymer concentration in the coacervate decreases with increasing salt concentration (see Figure 4 and 6). Surprisingly, however, the vertical shift factor $b_{s}$ increases slightly with salt concentration (Figure $8 \mathrm{~B}$ ). Liu and coworkers reported a similar trend for solid complexes in the vicinity of the gel point. ${ }^{32}$

We believe that this counterintuitive effect is due to the non-equilibrated structure of the gels. The viscoelastic properties below the gel point are controlled by the formation of growing clusters of microgels that eventually coalesce into a gel. The variation of terminal relaxation time of the coacervates with increasing level of association, i.e. decreasing concentration of salt, is intimately related to the dominant behavior of the cluster growth below the percolation threshold. ${ }^{54,58}$ Since this deviation happens to be particularly evident at low salt concentrations, strong aggregation leading to the non-equilibrated structures is plausible. The largest crosslinked cluster in the sample will also give the dominant contribution to the moduli, proportional to the concentration of these clusters. We then speculate that both the counterintuitive variation of the moduli with salt and the previous analysis of the doping level shown in Figure 5, highlighting the presence of salt in the coacervates in the form of counterions for 0.1 and $0.5 \mathrm{M}$ and with a large proportion of additional co-ions for $[\mathrm{NaCl}]>0.75 \mathrm{M}$, suggest a much broader impact of salt on the association behavior, affecting not only the lifetime of the stickers but also the architecture of the transient network through the number of binding sites per chain and their connectivity.

\subsection{Underwater probe tack}

\subsubsection{Adhesiveness in physiological conditions}

As highlighted by Winter and Mours, polymers close to the critical gel point are "extremely powerful adhesives" as they combine the wetting capability of liquids with the cohesive strength of solids. ${ }^{54}$ By probing adhesive properties of PDMS crosslinked to different degrees below and 
above the gel point, Zosel showed the highest adhesion energies are obtained with samples slightly into the gel regime, where the formation of stable fibrillar structures capable of maintaining a certain level of stress allowed bulk energy dissipation before failure. ${ }^{59}$ For underwater applications, coacervates offer key advantages over typical hydrophobic adhesives as they can wet immersed surfaces and remain immiscible with water. ${ }^{49,60,61}$ Yet, like any other adhesive, they must also be capable of sustain mechanical stress, for which they need to be close to the gel point.

Based on these insights, we investigated the underwater adhesiveness of two of our complex coacervates in media closely resembling body conditions, where they are close to the gel point (see Figure 7). The coacervate at $0.75 \mathrm{M}$, initially an injectable fluid, is studied after reinforcement upon immersion in $0.1 \mathrm{M}$ medium. Real-time evolution of its dynamic moduli during the salt switch are shown in Figure S6. The 0.1 M coacervate, on the other hand, is already close to its gel point and thus quite viscoelastic and compliant but not injectable. This sample was studied both in a $0.1 \mathrm{M} \mathrm{NaCl}$ solution (where no major salt switch is anticipated, see Figure S6) and in Phosphatebuffered saline (PBS). Figure 9 A and B show nominal stress-strain plots on lin-lin and log-lin scales from these probe tack experiments. The corresponding adhesion energies are presented in the bar chart in Figure $9 \mathrm{C}$, where error bars mark standard deviation from mean values form a minimum of 3 independently prepared samples. Underwater debonding of the adhesives was imaged in large deformations, shown in Figure 9 D.

The stress-strain behavior of these adhesives in the $0.1 \mathrm{M}$ medium is generically similar to that of soft, liquid-like adhesives and of confined viscoelastic fluids. ${ }^{59,62-64}$ All of the curves feature an initial peak in stress followed by a sharp fall. The slope of the stress versus strain curve is then reduced enabling the adhesive to maintain a certain level of stress up to very large deformations ( $\varepsilon_{\max } \geq 8$ and 25 for $0.75 \mathrm{M}$ and $0.1 \mathrm{M}$ samples, respectively; Figure 9B). In order for such deformations to be achieved both adhesives go through extensive fibrillation (Figure 9C), reminiscent of soft Pressure Sensitive Adhesives (PSAs) in air. However, our adhesives continue to soften up to cohesive failure with no indication of the strain hardening typically expected in PSAs and also seen in a soft, thermoresponsive underwater adhesive recently reported by our group. ${ }^{45,64,65}$ This was expected given that these adhesives are still in the sol state however close to the gel point (see Figure 7). ${ }^{59}$ 
A

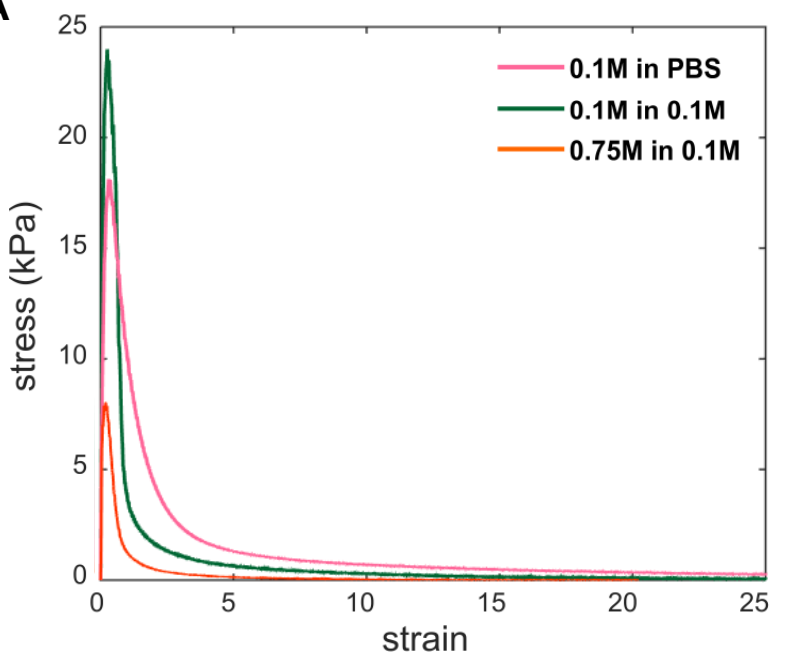

B

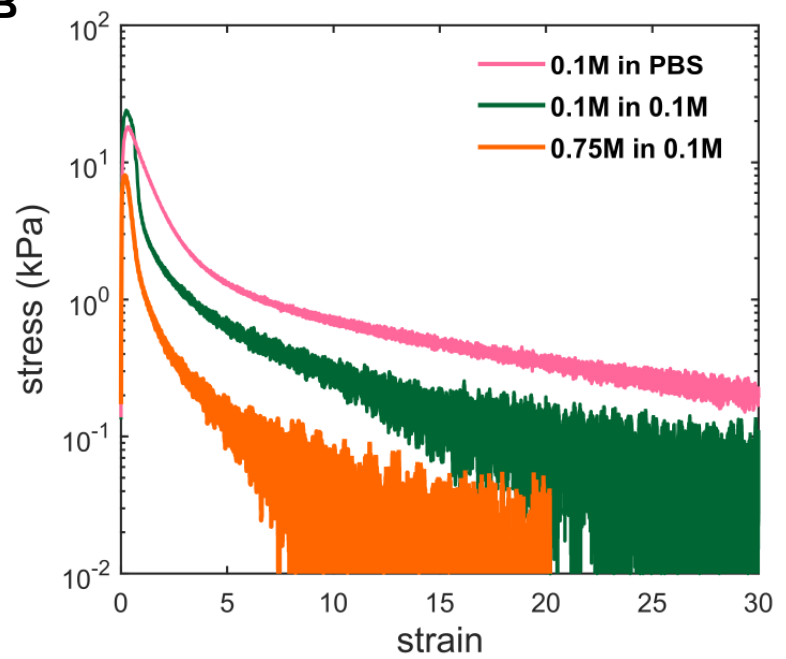

C

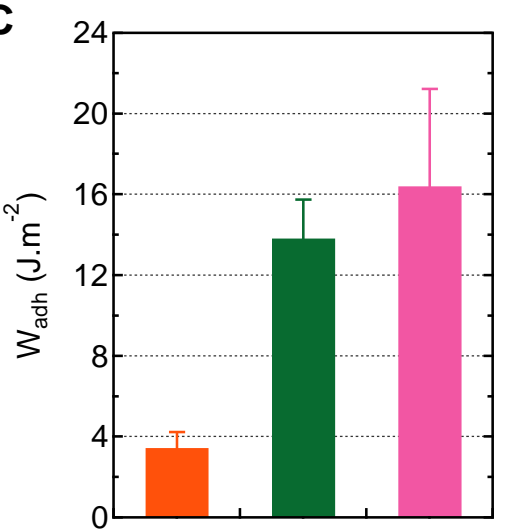

D
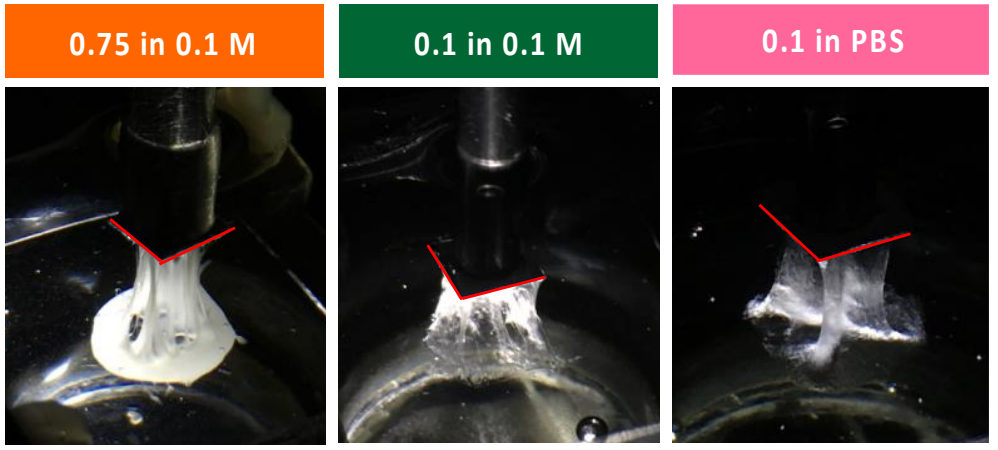

Figure 9. Nominal stress-strain curves from underwater probe tack experiments on 0.75 and 0.1 $M$ samples after 1 h of immersion in the medium specified in the legends. $(A)$ and $(B)$ present the data on lin-lin and log-lin scales, respectively. The corresponding adhesion energies and pictures of the adhesives during debonding under water are presented in $(C)$ and $(D)$, respectively. Error bars mark standard deviation from mean values from experiments on 3 independently prepared samples. The red lines added to the pictures mark the position of the probe. The color code is consistent in all figures.

The $0.75 \mathrm{M}$ coacervate was tested once reinforced via formation of extra macroion pairs upon injection of salt into the medium. The salt switch is indeed a slow, diffusion-controlled process taking several hours to complete, as seen in Figure S6, but the main enhancement in mechanical properties is realized in the first hour. This is also reflected in an immediate change from transparency to an opaque color (Figure 9D) which is plausibly due to the formation of microscopic pockets of water. ${ }^{37,40,49}$ In its hardened state, it has an adhesion energy of $3.5 \mathrm{~J} . \mathrm{m}^{-2}$ similar to those 
of Dompé and coworkers (2-6.5 J.m ${ }^{-2}$ ) under the same experimental conditions. ${ }^{37,40}$ However, it appears softer and less stretchable than the non-injectable $0.1 \mathrm{M}$ coacervate. Although this further confirms that a salt switch is a promising strategy to reinforce coacervate-based underwater adhesives, there seems to be an upper limit to the final mechanical properties which can be achieved with this strategy. We believe this is mainly due to the softening effect of the high equilibrium water content at high salt concentrations required for injectability.

The adhesiveness is clearly higher in the case of the $0.1 \mathrm{M}$ sample, where no major salt switch is expected (Figure S6) as further confirmed by its translucent appearance in Figure 9 D. This coacervate, close to its critical gel point with its two dynamic moduli ( $G^{\prime}$ and $G^{\prime \prime}$ ) almost equal and still frequency-dependent between 0.1-100 $\mathrm{rad}_{\mathrm{s}} \mathrm{s}^{-1}$ (see 3.2.1), is quite viscoelastic in large strain but stiffer than the $0.75 \mathrm{M}$ coacervate after a salt switch, with an adhesion energy of $13.8 \mathrm{~J}^{-\mathrm{m}^{-2}}$. To the best of our knowledge, this is the highest adhesion energy measured in underwater probe tack reported for a complex coacervate without an external trigger (salt, $\mathrm{pH}$, temperature), addition of divalent cations, or post curing reactions. This feature makes this adhesive a promising candidate for instant-stick solutions. Given the well-balanced properties of this coacervate, we will discuss it further, starting with experiments in PBS.

As seen in Figure 9, the adhesive appeared slightly softer (lower $\sigma_{\max }$ ) and more stretchable (higher $\varepsilon_{\max }$ ) in PBS with an adhesion energy of $16.4 \mathrm{~J}_{\mathrm{m}} \mathrm{m}^{-2}$, even higher than that in $0.1 \mathrm{M} \mathrm{NaCl}$. The gain in adhesion energy mainly comes from the higher stress level during the fibril stretching occurring after the peak stress $\sigma_{\max }$ (stress did not fall short of $100 \mathrm{~Pa}$ up to a strain of 40 , see Figure $9 \mathrm{~B}$ ). Although PBS is quite similar to the $0.1 \mathrm{M} \mathrm{NaCl}$ solution in terms of composition (with $0.137 \mathrm{M} \mathrm{NaCl}$ plus traces of other salts at concentrations below $3 \mathrm{mM}$ ) and $\mathrm{pH}(7.4)$, the presence of more $\mathrm{NaCl}$ and minority salts may very well lead to 1) uptake of more salt by the coacervate and 2) exchange of counter ions, possibly slightly modifying the structure of the gel, its average crosslinking density and bond lifetimes. This in turn mays lead to differences in fibril stress as we observe experimentally. One should keep in mind that the translucent appearance of the adhesive suggests a phase separated microstructure with a continuous phase of strong, polymer-rich regions (accounting for higher stress in large deformations) across a soft swollen matrix (corresponding to the lower peak stress).

\subsubsection{Mechanism of adhesion}


Given the viscoelastic nature of these adhesive coacervates, we also investigated the strain rate dependence of their mechanical response, as shown in Figure $10 \mathrm{~A}$ and B for the $0.1 \mathrm{M}$ sample. The adhesion energies are plotted as a function strain rate in Figure $10 \mathrm{C}$ and show a significant strain rate dependence, a hallmark of the type of bulk viscoelastic dissipation observed in soft adhesives. ${ }^{66,67} \mathrm{We}$ also observe a bulk fingering instabilities following the homogeneous deformation of the layer at higher strain rates consistent with previous studies on viscoelastic fluids ${ }^{67-69}$ Tests at higher strain rates result in a stiffer and more elastic response with a plateau in stress beginning to appear in large deformations at $2 \mathrm{~s}^{-1}$, causing a much higher energy dissipation in the bulk of the material prior to cohesive failure.

A

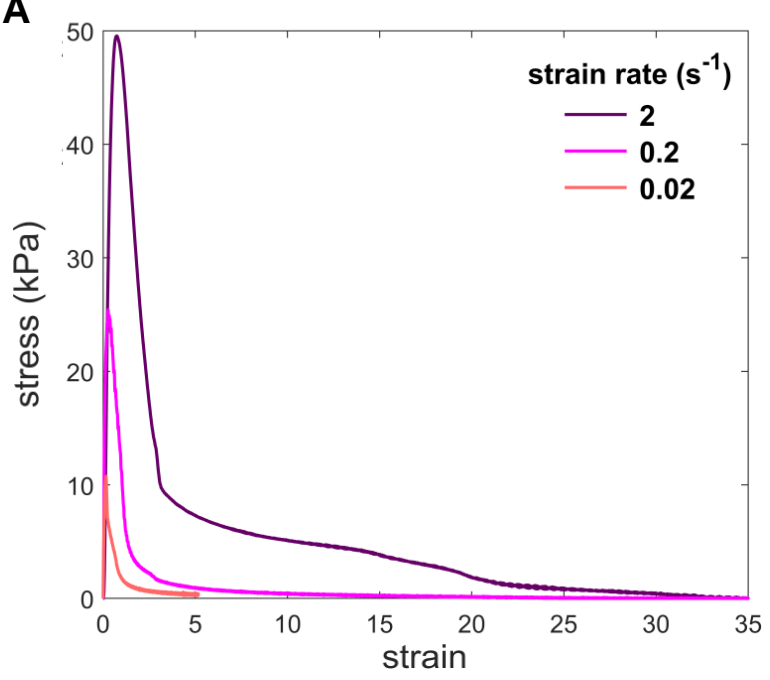

B

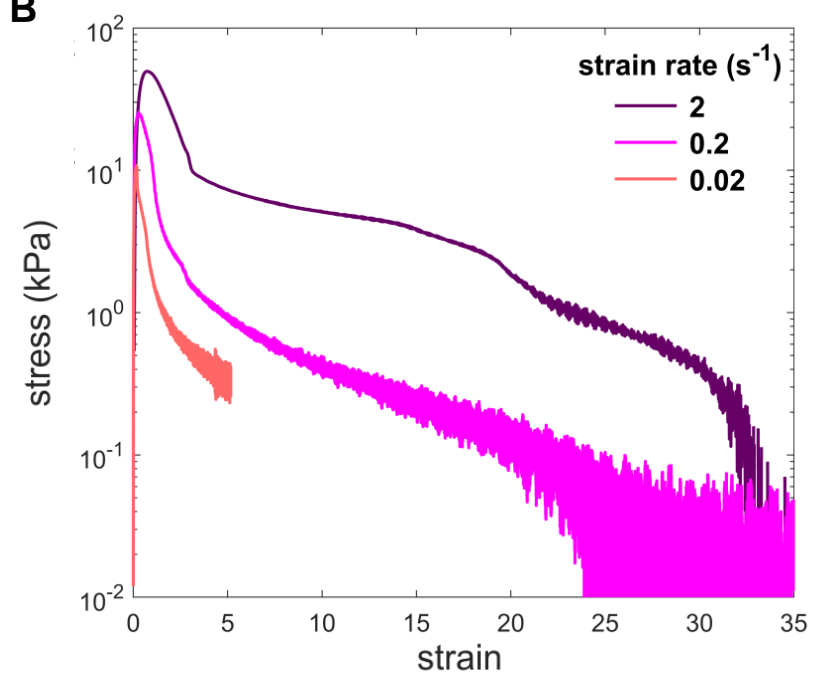

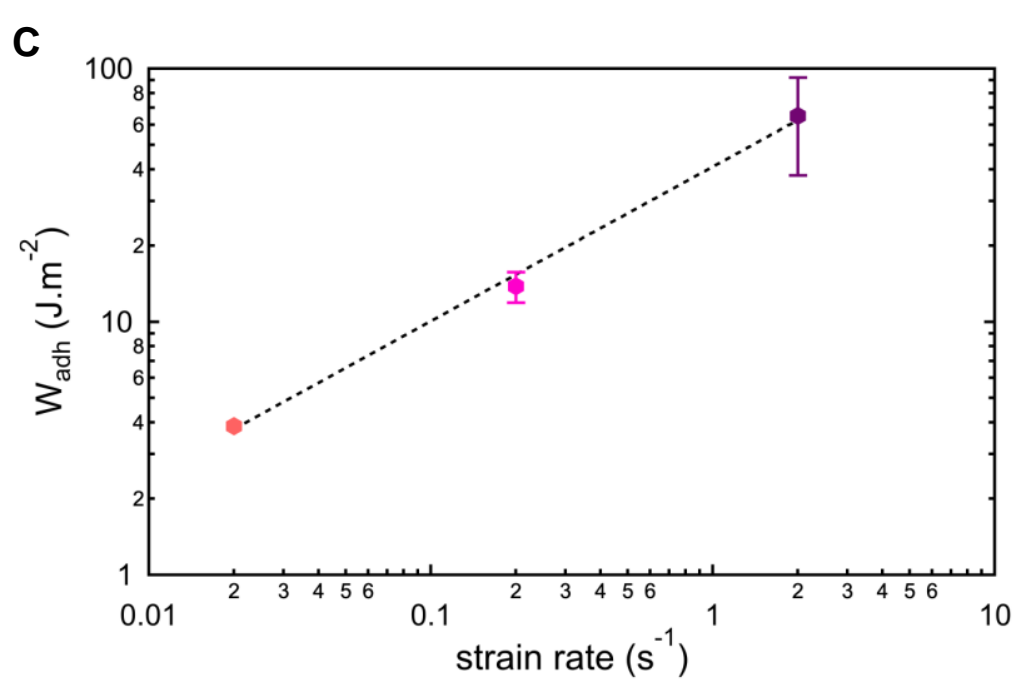

Figure 10. Nominal stress-strain curves from underwater probe tack experiments at different nominal strain rates on $0.1 \mathrm{M}$ coacervate after $1 \mathrm{~h}$ of immersion in $0.1 \mathrm{M} \mathrm{NaCl}$. (A) and (B) 
present the data on lin-lin and log-lin scales, respectively. The adhesion energies as a function of nominal strain rate are plotted in $(C)$ in a log-log scale. The dashed line corresponds to the best fit to the data with an expression of the type $W_{a d h} \propto \dot{\varepsilon}^{a}$. The best fit gives $a=0.61$. Error bars are standard deviation from mean values (3 replicates from independently prepared samples).

The color code is consistent in all figures.

Figure $10 \mathrm{C}$ plots $W_{a d h}$ against $\dot{\varepsilon}$ in a $\log -\log$ scale. The best fit $\left(\mathrm{r}^{2}=99.8\right)$ to the data indicates a power-law behavior, $W_{a d h} \propto \dot{\varepsilon}^{a}$, with the exponent $a=0.6$. Power law exponents of typical solid- like adhesives fall in the range of $0.1-0.3$, with larger exponents signifying a more viscous response in the material, as is the case with our coacervates. ${ }^{70,71}$ Again, this is consistent with the fact that these adhesives are not in the gel regime at this salt concentration. We note that a strain rate of $2 \mathrm{~s}^{-1}$ corresponds to a probe velocity of $1 \mathrm{~mm} \cdot \mathrm{s}^{-1}$, reasonably close to the manual retraction

of a finger. The underwater $W_{a d h}$ in these realistic conditions $\left(65.2 \mathrm{~J} . \mathrm{m}^{-2}\right)$ amounts to those of Post$\mathrm{It}^{\circledR}$ notes and soft healthcare adhesives in air.

This coacervate is too stiff to be injectable but is sufficiently soft and compliant $\left(G^{\prime}<100 \mathrm{kPa}\right.$ at $1 \mathrm{~Hz}$, consistent with Dahlquist's criterion) to be shaped like a paste and easily squeezed. It is then easily capable of making intimate contact with surfaces under a light pressure and the large value of the loss modulus helps to dissipate considerable energy during debonding. ${ }^{67,71,72}$ It can be directly applied under water before contact with the probe without a reduction in adhesion energy, as shown in Figure S7 in the SI. Another advantage of the $0.1 \mathrm{M}$ coacervate for potential biomedical applications is that it eliminates the need for a switch, meaning that it can be easily formed, for instance, into a thin layer and used as an underwater adhesive tape or patch. The insolubility of complex coacervates in aqueous environments, resulting in a stable water content over time, is one of the distinct advantages giving them an edge over other swellable materials for underwater adhesion. ${ }^{4,49}$

\section{Discussion}

Among various parameters which can be potentially used to tune the mechanical properties of complex coacervates, the molecular weight of the polyelectrolytes has not received due attention. Understanding the significance of this parameter requires establishing clear links between the composition of these soft materials and their structure and properties. 
It is thus insightful to start with the role of molecular weight in the equilibrium composition of complex coacervates. As previously highlighted by Spruijt and coworkers as well as Wang and Schlenoff, the CSC shifts towards smaller values for lower DPs in matched complex coacervates (i.e. having roughly the same DPs). ${ }^{6,10}$ This stems from the more soluble character of smaller molecular weights due to the gain in entropy of mixing. Concurrently and for the same reason, the two-phase region of the phase diagram shrinks, diminishing the polymer content in the coacervate phase or, analogously, increasing swelling. This sets a limit to the molecular weight below which the mechanical properties become too weak.

The molecular weight dependence of the equilibrium water content in the coacervate has previously been studied for relatively hydrophilic polyelectrolytes at negligible salt concentrations. ${ }^{10}$ There is a lower limit, around $60-70 \mathrm{wt} \%$, to the attainable water content for DPs above 100. Polyelectrolytes with very small DPs $(<10)$ are nearly fully soluble with the water content approaching $100 \mathrm{wt} \%$. Between these limits, the equilibrium water content varies sharply with molecular weight. Given the similarity of the water content in our system (56 wt \% in the 0.1 M sample, see Figure 4) to those reported for hydrophilic complex coacervates in the literature justifies our choice of targeting DPs around 100. ${ }^{10,24,33}$ Higher salt concentrations then lead to swelling which in turn softens the coacervates.

This was clearly manifested in the rheological behavior of our coacervates prepared at different salt concentrations, with liquid-like injectable samples at high salt concentrations and increasingly viscoelastic materials towards low salt concentrations (see Figure 7). We are in fact the first to report the sol-gel transition in complex coacervates from polyelectrolytes with such small DPs. This allowed us to tune the sol-gel transition, where stickiness is optimized, close to physiological salt concentrations. We note that currently reported sol-gel transitions occur at concentration of salt exceeding 0.6 M, characteristic of the polyelectrolytes with DPs > 500. . $^{6,32,33,40}$

We believe that the semi-dilute unentangled regime seen in rheology (see Figure 8) mainly arises from the use of such small DPs and has less to do with the salt or the nature of the polyelectrolytes. Larger molecular weights of the same polyelectrolytes used in this work have not been studied before, yet we expect our hypothesis should remain true for systems of similar water contents, i.e. based on polyelectrolytes of similar hydrophilicity. In other terms, reducing the salt content will not entail an elastic plateau in rheology if the molecular weights fall short of the 
entangled regime. As extensively explained before, the sole effect of removing salt is increasing internal friction against chain mobility by raising the energy barrier of dissociation.

Therefore, targeting DPs around 100 with hydrophilic polyelectrolytes offers a triple advantage. First, this is the lowest DP where the minimum equilibrium water content, corresponding to the best mechanical properties, can be achieved. As discussed in details in one of our previous studies, increasing the initial polymer concentration is not an effective strategy to lower the water content; it simply increases the relative volume of the coacervate phase without modifying its equilibrium composition. ${ }^{34}$ Second, the molecular weight is not high enough to form entanglements, and therefore the coacervates remain in the semi-dilute unentangled regime, which can be an asset in designing injectable underwater adhesives. Last but not least, sol-gel transition occurs at salt concentrations more practical and convenient for potential biomedical applications.

It is also important to discuss the tradeoff between the injectability and the final mechanical properties of the $0.75 \mathrm{M}$ coacervate after salt switch as opposed to the sample already prepared at $0.1 \mathrm{M} \mathrm{NaCl}$ (see Figure 9). Even after long immersion times $(14 \mathrm{~h})$, the dynamic moduli of the initially liquid-like coacervate did not reach the same level as those of the $0.1 \mathrm{M}$ sample (Figure S7). This is probably mainly because the polymer content remains unchanged during the salt switch (some $10 \mathrm{wt} \%$ lower than the $0.1 \mathrm{M}$ coacervate), even after complete removal of salt. The structure and composition of the coacervate is therefore not equilibrated. Another possibility is the shrinkage of the sample and a weaker interface with the probes, although we believe this to be less likely as no macroscopic volume change was visible.

As underwater adhesives, both coacervates eventually appear generically similar with extensive fibrillation and bulk energy dissipation, but the gel-like 0.1 M sample is 3-4 times stronger in terms of adhesion energy. This non-injectable sample can be used as an instant-stick solution offering the possibility of direct contact under water (see Figure S7) and easy removal at short time scales ( $~ 5 \mathrm{~min}$, see Figure S8). Based on the mechanical behavior of this coacervate and partly inspired by the molecular insights from the work of Hamad and coworkers, ${ }^{24}$ we suggest the nanostructure shown in Figure 11. We expect this picture to remain self-similar for samples at higher salt concentrations, with the water-rich regions becoming more predominant as the macroion pairs are screened. 


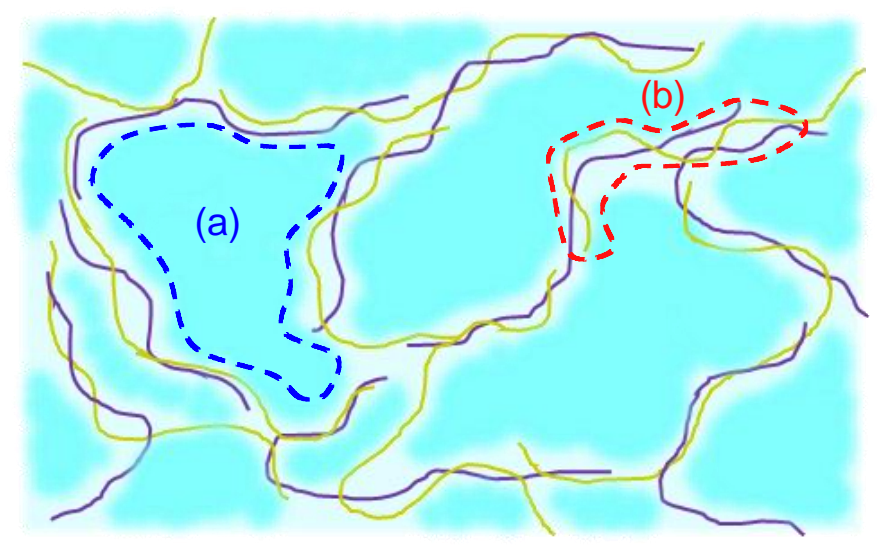

Figure 11. The plausible nanostructure deduced from Time-Salt Superposition and underwater probe tack experiments for nearly matched complex coacervates of PAMPS and PMADAP with DP around 100. The regions within the blue and red dashed lines represent (a) soft, water-rich regions and (b) strong, polymer-rich domains, respectively.

If cohesive failure is acceptable, i.e. when clean removal from the surfaces is not required, we propose tuning the water content and viscoelastic properties of complex coacervates by controlling the degree of polymerization rather than using high concentrations of salt, which can be harmful to body tissues in potential applications. In fact, the adhesive properties of our coacervates are characteristic of fluid-like adhesives, simply because they remain in the sol vicinity of the sol-gel transition. Therefore, making yet stronger adhesives under physiological conditions requires that the transition occur at salt concentrations slightly higher than that of the body, around $0.2 \mathrm{M}$. It may be interesting to target slightly higher DPs, in the range of 200-300. As such, the adhesive will end up in the gel vicinity of the transition under physiological conditions, thereby showing maximal adhesiveness. ${ }^{59}$ However, achieving strain-hardening and adhesive removal remain open issues without formation of entanglements. The novel insights from this work pave the road towards safe underwater adhesives for potential biomedical applications.

\section{Conclusions}

Fine-tuning underwater adhesiveness of complex coacervates requires close control over their composition. Instead of focusing on high molecular weight polyelectrolytes which would demand high salt concentrations to make liquid-like coacervates, we have used relatively short, hydrophilic polyelectrolytes with DPs around 100. This strategy offers several merits including proximity to 
the sol-gel transition and thus being in a viscoelastic, sticky state under physiological salt concentrations. The injectable coacervate we prepared at $0.75 \mathrm{M}$ salt can be mechanically reinforced via a salt switch into physiological conditions, but there is still a tradeoff between injectability and final mechanical properties. However, we also designed a coacervate in $0.1 \mathrm{M}$ salt, which is just above the gel point and contains a minimal amount of water (56 wt \%) stable over time. This coacervate features a remarkable adhesion energy of $65 \mathrm{~J} . \mathrm{m}^{-2}$ in physiological salt concentration. Although not injectable, this sticky coacervate can be directly applied under water in the form of adhesive tapes or patches. Our findings highlight the untapped potentials of coacervates from relatively low molecular weight polyelectrolytes in developing underwater sticky materials potentially useful for bonding biological tissues.

6. Associated content

Synthetic details, ${ }^{1} \mathrm{H}-\mathrm{NMR}$, TGA validation and calculations, swelling, TSS calculations, salt switch via linear rheology, direct underwater contact as well as contact time are given in the Supporting Information.

7. Funding

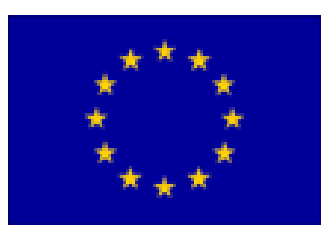

This work was part of a BioSmartTrainee Network project. The project received funding from the European Union's Horizon 2020 research and innovation program under the Marie Sklodowska-Curie grant agreement no. 642861.

\section{Acknowledgements}

The authors would also like to warmly thank Dr. Guylaine Ducouret for assistance with rheology experiments, and Dr. Marco Dompé from Wageningen University and Professor Marleen Kamperman from University of Groningen for fruitful discussions. 
9. References

(1) Srivastava, S.; Tirrell, M. V. Polyelectrolyte Complexation. In Advances in Chemical Physics; Stuart A. Rice, A. D. D., Ed.; Wiley, 2016; Vol. 161, pp 499-544.

(2) Nakashima, K. K.; Vibhute, M. A.; Spruijt, E. Biomolecular Chemistry in Liquid Phase Separated Compartments. Front. Mol. Biosci. 2019, 6 (April).

(3) Stewart, R. J.; Ransom, T. C.; Hlady, V. Natural Underwater Adhesives. J. Polym. Sci. Part B Polym. Phys. 2011, 49 (11), 757-771.

(4) Stewart, R. J.; Wang, C. S.; Song, I. T.; Jones, J. P. The Role of Coacervation and Phase Transitions in the Sandcastle Worm Adhesive System. Adv. Colloid Interface Sci. 2017, 239, 88-96.

(5) Stewart, R. J.; Wang, C. S.; Shao, H. Complex Coacervates as a Foundation for Synthetic Underwater Adhesives. Adv. Colloid Interface Sci. 2011, 167 (1-2), 85-93.

(6) Wang, Q.; Schlenoff, J. B. The Polyelectrolyte Complex/Coacervate Continuum. Macromolecules 2014, 47.

(7) Turgeon, S. L.; Schmitt, C.; Sanchez, C. Protein-Polysaccharide Complexes and Coacervates. Curr. Opin. Colloid Interface Sci. 2007, 12 (4-5), 166-178.

(8) Schlenoff, J. B.; Yang, M.; Digby, Z. A.; Wang, Q. Ion Content of Polyelectrolyte Complex Coacervates and the Donnan Equilibrium. Macromolecules 2019, 52 (23), 91499159.

(9) Sing, C. E. Development of the Modern Theory of Polymeric Complex Coacervation. Adv. Colloid Interface Sci. 2017, 239, 2-16.

(10) Spruijt, E.; Westphal, A. H.; Borst, J. W.; Cohen Stuart, M. A.; Van Der Gucht, J. Binodal Compositions of Polyelectrolyte Complexes. Macromolecules 2010, 43 (15), 6476-6484.

(11) Fu, J.; Schlenoff, J. B. Driving Forces for Oppositely Charged Polyion Association in Aqueous Solutions: Enthalpic, Entropic, but Not Electrostatic. J. Am. Chem. Soc. 2016, 138 (3), 980-990.

(12) Shen, K.; Wang, Z. G. Polyelectrolyte Chain Structure and Solution Phase Behavior. Macromolecules 2018, 51 (5), 1706-1717.

(13) Andreev, M.; Prabhu, V. M.; Douglas, J. F.; Tirrell, M.; De Pablo, J. J. Complex Coacervation in Polyelectrolytes from a Coarse-Grained Model. Macromolecules 2018, 51 (17), 6717-6723. 
(14) Zhang, P.; Alsaifi, N. M.; Wu, J.; Wang, Z. G. Salting-Out and Salting-In of Polyelectrolyte Solutions: A Liquid-State Theory Study. Macromolecules 2016, 49 (24), 9720-9730.

(15) Radhakrishna, M.; Basu, K.; Liu, Y.; Shamsi, R.; Perry, S. L.; Sing, C. E. Molecular Connectivity and Correlation Effects on Polymer Coacervation. Macromolecules 2017, 50 (7), 3030-3037.

(16) Lytle, T. K.; Sing, C. E. Transfer Matrix Theory of Polymer Complex Coacervation. Soft Matter 2017, 13 (39), 7001-7012.

(17) Zhang, P.; Shen, K.; Alsaifi, N. M.; Wang, Z. G. Salt Partitioning in Complex Coacervation of Symmetric Polyelectrolytes. Macromolecules 2018, 51 (15), 5586-5593.

(18) Lu Li, Samanvaya Srivastava, Marat Andreev, Amanda B. Marciel, Juan J. de Pablo, and M. V. T. Phase Behavior and Salt Partitioning in Polyelectrolyte Complex Coacervates. Macromolecules 2018, 51 (8), 2988-2995.

(19) Salehi, A.; Larson, R. G. A Molecular Thermodynamic Model of Complexation in Mixtures of Oppositely Charged Polyelectrolytes with Explicit Account of Charge Association/Dissociation. Macromolecules 2016, 49 (24), 9706-9719.

(20) J. T. G. Overbeek, M. J. V. Phase Separation in Polyelectrolyte Solutions. Theory of Complex Coacervation. J. Cell. Comp. Physiol. 1957, 49, 7-26.

(21) Gucht, J. van der; Spruijt, E.; Lemmers, M.; Cohen Stuart, M. A. Polyelectrolyte Complexes: Bulk Phases and Colloidal Systems. J. Colloid Interface Sci. 2011, 361 (2), 407-422.

(22) Rumyantsev, A. M.; Zhulina, E. B.; Borisov, O. V. Complex Coacervate of Weakly Charged Polyelectrolytes: Diagram of States. Macromolecules 2018, 51 (10), 3788-3801.

(23) Rubinstein, M.; Semenov, A. N. Dynamics of Entangled Solutions of Associating Polymers. Macromolecules 2001, 34 (4), 1058-1068.

(24) Hamad, F. G.; Chen, Q.; Colby, R. H. Linear Viscoelasticity and Swelling of Polyelectrolyte Complex Coacervates. Macromolecules 2018, 51 (15), 5547-5555.

(25) Spruijt, E.; Sprakel, J.; Lemmers, M.; Stuart, M. A. C.; Van Der Gucht, J. Relaxation Dynamics at Different Time Scales in Electrostatic Complexes: Time-Salt Superposition. Phys. Rev. Lett. 2010, 105 (20), 1-4.

(26) Yang, M.; Shi, J.; Schlenoff, J. B. Control of Dynamics in Polyelectrolyte Complexes by 
Temperature and Salt. Macromolecules 2019, 52 (5), 1930-1941.

(27) Spruijt, E.; Van Den Berg, S. A.; Cohen Stuart, M. A.; Van Der Gucht, J. Direct

Measurement of the Strength of Single Ionic Bonds between Hydrated Charges. ACS Nano 2012, 6 (6), 5297-5303.

(28) Spruijt, E.; Cohen Stuart, M. A.; Van Der Gucht, J. Linear Viscoelasticity of Polyelectrolyte Complex Coacervates. Macromolecules 2013, 46 (4), 1633-1641.

(29) Liu, Y.; Winter, H. H.; Perry, S. L. Linear Viscoelasticity of Complex Coacervates. Adv. Colloid Interface Sci. 2017, 239, 46-60.

(30) Fu, J.; Fares, H. M.; Schlenoff, J. B. Ion-Pairing Strength in Polyelectrolyte Complexes. Macromolecules 2017, 50 (3), 1066-1074.

(31) Marciel, A. B.; Srivastava, S.; Tirrell, M. V. Structure and Rheology of Polyelectrolyte Complex Coacervates. Soft Matter 2018, 14 (13), 2454-2464.

(32) Liu, Y.; Momani, B.; Winter, H. H.; Perry, S. L. Rheological Characterization of Liquidto-Solid Transitions in Bulk Polyelectrolyte Complexes. Soft Matter 2017, 13 (40), 7332 7340 .

(33) Shull, K. R.; Jiang, Z.; Wang, Q.; Keshavarz, B.; Chen, Y.; Sadman, K. Influence of Hydrophobicity on Polyelectrolyte Complexation. Macromolecules 2017, 50 (23), 94179426.

(34) Dompe, M.; Vahdati, M.; Ligten, F. Van; Cedano-Serrano, F. J.; Hourdet, D.; Creton, C.; Zanetti, M.; Bracco, P.; Gucht, J. Van Der; Kodger, T.; Kamperman, M. Enhancement of the Adhesive Properties by Optimizing the Water Content in PNIPAM-Functionalized Complex Coacervates. ACS Appl. Polym. Mater. 2020.

(35) Schlenoff, J. B.; Rmaile, A. H.; Bucur, C. B. Hydration Contributions to Association in Polyelectrolyte Multilayers and Complexes: Visualizing Hydrophobicity. J. Am. Chem. Soc. 2008, 130 (41), 13589-13597.

(36) Rubinstein, Michael, R. H. C. Polymr Physics; Oxford, 2003.

(37) Dompé, M.; Cedano-Serrano, F. J.; Vahdati, M.; Sidoli, U.; Heckert, O.; Synytska, A.; Hourdet, D.; Creton, C.; van der Gucht, J.; Kodger, T.; Kamperman, M. Tuning the Interactions in Multiresponsive Complex Coacervate-Based Underwater Adhesives. Int. J. Mol. Sci. 2020, 21 (1).

(38) Sadman, K.; Delgado, D. E.; Won, Y.; Wang, Q.; Gray, K. A.; Shull, K. R. Versatile and 
High-Throughput Polyelectrolyte Complex Membranes via Phase Inversion. ACS Appl. Mater. Interfaces 2019, 11, 16018-16026.

(39) Jones, J. P.; Sima, M.; O’Hara, R. G.; Stewart, R. J. Water-Borne Endovascular Embolics Inspired by the Undersea Adhesive of Marine Sandcastle Worms. Adv. Healthc. Mater. 2016, 5 (7), 795-801.

(40) Dompé, M.; Cedano-Serrano, F. J.; Vahdati, M.; van Westerveld, L.; Hourdet, D.; Creton, C.; van der Gucht, J.; Kodger, T.; Kamperman, M. Underwater Adhesion of Multiresponsive Complex Coacervates. Adv. Mater. Interfaces 2019, 1901785.

(41) Kohei MURAKAWA, Daniel R. KING, Taolin SUN, Honglei GUO, T. Kurokawa. and J. P. G. Polyelectrolyte Complexation via Viscoelastic Phase Separation Results in Tough and Self-Recovering Porous Hydrogels. Mater. Chem. B 2019.

(42) Hofman, A. H.; van Hees, I. A.; Yang, J.; Kamperman, M. Bioinspired Underwater Adhesives by Using the Supramolecular Toolbox. Adv. Mater. 2018, 30 (19), 1704640.

(43) Durand, A.; Hourdet, D. Synthesis and Thermoassociative Properties in Aqueous Solution of Graft Copolymers Containing Poly ( N -Isopropylacrylamide ) Side Chains. Polymer (Guildf). 1999, 40, 4941-4951.

(44) Hourdet, D.; Petit, L.; Karakasyan, C. Synthesis of Graft Polyacrylamide with Responsive Self-Assembling Properties in Aqueous Media. 2007, 48, 7098-7112.

(45) Vahdati, M.; Ducouret, G.; Creton, C.; Hourdet, D. Thermally Triggered Injectable Underwater Adhesives. 2020, 1900653, 1-7.

(46) Sudre, G.; Olanier, L.; Tran, Y.; Hourdet, D.; Creton, C. Reversible Adhesion between a Hydrogel and a Polymer Brush. Soft Matter 2012, 8 (31), 8184-8193.

(47) Macron, J.; Bresson, B.; Tran, Y.; Hourdet, D.; Creton, C. Equilibrium and Out-ofEquilibrium Adherence of Hydrogels against Polymer Brushes. Macromolecules 2018, 51, 7556-7566.

(48) Cedano-Serrano, F. J.; Sidoli, U.; Synytska, A.; Tran, Y.; Hourdet, D.; Creton, C. From Molecular Electrostatic Interactions and Hydrogel Architecture to Macroscopic Underwater Adherence. Macromolecules 2019, 52 (10), 3852-3862.

Dompé, M.; Cedano-Serrano, F. J.; Heckert, O.; van den Heuvel, N.; van der Gucht, J.; Tran, Y.; Hourdet, D.; Creton, C.; Kamperman, M. Thermoresponsive Complex Coacervate-Based Underwater Adhesive. Adv. Mater. 2019, 1808179, 1808179. 
(50) Reisch, A.; Tirado, P.; Roger, E.; Boulmedais, F.; Collin, D.; Voegel, J. C.; Frisch, B.; Schaaf, P.; Schlenoff, J. B. Compact Saloplastic Poly(Acrylic Acid)/Poly(Allylamine) Complexes: Kinetic Control over Composition, Microstructure, and Mechanical Properties. Adv. Funct. Mater. 2013, 23 (6), 673-682.

(51) Shamoun, R. F.; Reisch, A.; Schlenoff, J. B. Extruded Saloplastic Polyelectrolyte Complexes. Adv. Funct. Mater. 2012, 22 (9), 1923-1931.

(52) Schlenoff, J. B. Site-Specific Perspective on Interactions in Polyelectrolyte Complexes: Toward Quantitative Understanding. J. Chem. Phys. 2018, 149 (16).

(53) Calvo, E. J.; Wolosiuk, A. Donnan Permselectivity in Layer-by-Layer Self-Assembled Redox Polyelectrolye Thin Films. J. Am. Chem. Soc. 2002, 124 (28), 8490-8497.

(54) Winter, H. H.; Mours, M. Rheology of Polymers Near Liquid-Solid Transitions. Adv. Polym. Sci. 1997, 134, 165-234.

(55) Henning Winter, H. The Occurrence of Self-Similar Relaxation in Polymers. J. Non. Cryst. Solids 1994, 172-174 (PART 2), 1158-1167.

(56) Colby, R. H. Structure and Linear Viscoelasticity of Flexible Polymer Solutions: Comparison of Polyelectrolyte and Neutral Polymer Solutions. Rheol. Acta 2010, 49 (5), 425-442.

(57) Ali, S.; Prabhu, V. Relaxation Behavior by Time-Salt and Time-Temperature Superpositions of Polyelectrolyte Complexes from Coacervate to Precipitate. Gels 2018, 4 $(1), 11$.

(58) Rubinstein, M.; Semenov, A. N. Thermoreversible Gelation in Solutions of Associating Polymers. 2. Linear Dynamics. Macromolecules 1998, 31 (4), 1386-1397.

(59) Zosel, A. Effect of Cross-Linking on Tack and Peel Strength of Polymers. J. Adhes. 1991, 34 (1-4), 201-209.

(60) Shao, H.; Bachus, K. N.; Stewart, R. J. A Water-Borne Adhesive Modeled after the Sandcastle Glue of P. Californica. Macromol. Biosci. 2009, 9 (5), 464-471.

(61) Shao, H.; Stewart, R. J. Biomimetic Underwater Adhesives with Environmentally Triggered Setting Mechanisms. Adv. Mater. 2010, 22 (6), 729-733.

(62) Poivet, S.; Nallet, F.; Gay, C.; Fabre, P. Cavitation-Induced Force Transition in Confined Viscous Liquids under Traction. Europhys. Lett. 2003, 62 (2), 244-250.

(63) Poivet, S.; Nallet, F.; Gay, C.; Teisseire, J.; Fabre, P. Force Response of a Viscous Liquid 
in a Probe-Tack Geometry: Fingering versus Cavitation. Eur. Phys. J. E 2004, 15 (2), $97-$ 116.

(64) Deplace, F.; Carelli, C.; Mariot, S.; Retsos, H.; Chateauminois, A.; Ouzineb, K.; Creton, C. Fine Tuning the Adhesive Properties of a Soft Nanostructured Adhesive with Rheological Measurements. J. Adhes. 2009, 85 (1), 18-54.

(65) Shull, K. R.; Creton, C. Deformation Behavior of Thin, Compliant Layers under Tensile Loading Conditions. J. Polym. Sci. Part B Polym. Phys. 2004, 42 (22), 4023-4043.

(66) Creton, C. 50th Anniversary Perspective: Networks and Gels: Soft but Dynamic and Tough. Macromolecules 2017, 50 (21), 8297-8316.

(67) Creton, C.; Ciccotti, M. Fracture and Adhesion of Soft Materials: A Review. Reports Prog. Phys. 2016, 79 (4), 46601.

(68) Lakrout, H.; Sergot, P.; Creton, C. Direct Observation of Cavitation and Fibrillation in a Probe Tack Experiment on Model Acrylic Pressure-Sensitive-Adhesives. J. Adhes. 1999, 69 (3-4), 307-359.

(69) Lakrout, H.; Creton, C.; Ahn, D.; Shull, K. R. Influence of Molecular Features on the Tackiness of Acrylic Polymer Melts. Macromolecules 2001, 34 (21), 7448-7458.

(70) Gent, A. N.; Petrich, R. P. A Dhesion of Viscoelastic Materials to Rigid Substrates. Proc. Roy. Soc. A 1969, 310, 433-448.

(71) Gent, A. N.; Schultz, J. Effect of Wetting Liquids on the Strength of Adhesion of Viscoelastic Materials. J. Adhes. 1972, 3 (4), 281-294.

(72) Gdalin, B. E.; Bermesheva, E. V.; Shandryuk, G. A.; Feldstein, M. M. Effect of Temperature on Probe Tack Adhesion: Extension of the Dahlquist Criterion of Tack. J. Adhes. 2011, 87 (2), 111-138. 
For Table of Contents Only
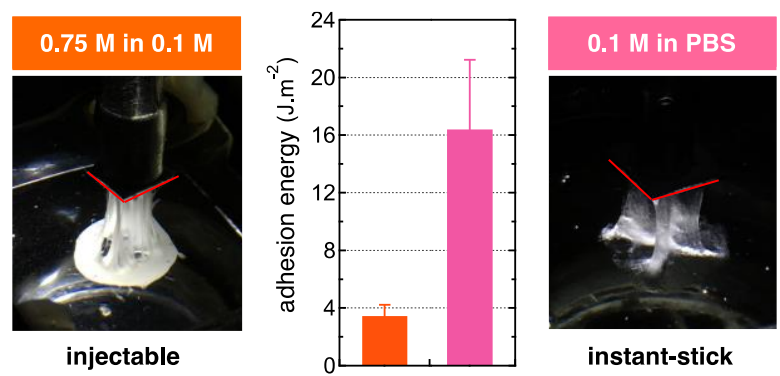

instant-stick 